\title{
On Asymptotic Behaviour of the Spectra of a One-Dimensional Hamiltonian with a Certain Random Coefficient
}

By

Shin'ichi KOTANI*

\section{§o. Hntroduction}

Let $\{Q(x)\}$ be a random process with stationary independent increments. We consider a second order differential operator $L$ defined by

$$
L \varphi=-\frac{d \frac{d \varphi}{d x}-\varphi d Q(x)}{d x} .
$$

Let $N(\lambda, I)$ be the number of eigenvalues not exceeding $\lambda$ for a certain boundary value problem of the operator $L$ in the interval $I$. We define the spectral distribution function of $L$ by

$$
N(\lambda)=\lim _{|I| \rightarrow \infty} \frac{1}{|I|} N(\lambda, I),
$$

if it exists, where $|I|$ is the length of $I$.

The operator $L$ has been used as a Schrödinger operator describing a motion of an electron in a one-dimensional random array of atoms (cf. M. Lax-J. C. Phillips [1], I. M. Lifšic [2]). We are concerned with the study of asymptotic properties of $N(\lambda)$ at the edges of the support. One interest is in making clear the influences caused by the randommess of potentials. One of them is the exponential decay of $N(\lambda)$ at the left edge, which was shown by many authors for various potentials (cf. H. L. Frisch-S. P. Lloyd [3], M. M. Benderskii-L. A. Pastur [4], [5], T. P. Eggarter [6]). L. A. Pastur [7] is a survey written mainly from mathematical points of view and gives us good informations about the problems arising

* Department of Mathematics, Kyoto University, Kyoto 606, Japan. 
from random differential equations.

The author, suggested by H. L. Frisch-S. P. Lloyd [3], has succeeded in developing their results to obtain a sharper estimate of $N(\lambda)$ at the left end point. The purpose of the present paper is to give a complete proof of a formula obtained by H. L. Frisch-S. P. Lloyd [3] for potentials belonging to a slightly wider class and to obtain the estimate of $N(\lambda)$ by analyzing the formula on the pure imaginary axis. In the proof of the formula, the author was given valuable suggestions from $M$. Fukushima-S. Nakao [8]. Further the author would like to remark that S. Nakao [9] has obtained satisfactory results in several dimensional case by making use of the result of Donsker-Varadhan on "Wiener sausage".

Now, we explain the content of this paper. In $\S 1$, as a preparation for the latter sections, we shall prove some properties relating to the zeros of eigenfunctions of a generalized differential operator

$$
\frac{d \frac{d \varphi}{d x}-\varphi d Q(x)}{d M(x)} .
$$

In $\S 2$, we shall prove the ergodic property of the solution of a Ricatti equation with a random coefficient

$$
d z(x)=\left(z(x)^{2}+\lambda\right) d x-d Q(x),
$$

where $\lambda>0$ and $Q$ is a process with stationary independent increments [Theorem 2.5]. With the help of this theorem, the Rice formula and the Frisch-Lloyd formula will be proved [Corollary 2.6 and 2.8]. In $\S 3$, we restrict ourselves to the case when the process $Q$ is increasing and express the spectra $N(\lambda)$ in a simpler form [Theorem 3.2]. In $\S 4$, applying this form, we shall obtain the main result for the asymptotic behaviour of $N(\lambda)$ at the origin [Theorem 4.7]. In $\S 5$, we shall give an expression of the spectral distribution $N(\lambda)$ of an equation

$$
\frac{d}{d M} \frac{d \varphi}{d x}=-\lambda \varphi
$$

where $M$ is an increasing process with stationary independent increments. In $\S 6$, we shall give a comment on the spectral distribution of an equation defined on the whole line $\boldsymbol{R}^{1}$. 
The author wishes to express thanks to Prof. M. Fukushima for teaching him the problem and to Prof. S.Watanabe for his constant attention to the present work.

\section{$\S 1$. On the Behaviour of Zeros of Eigenfunctions of Generalized Second Order Differential Equations}

First of all, let us introduce necessary notations and terminologies. Let $[a, b]$ be a finite closed interval. We denote $f \in V[a, b]$, if $f$ consists of a function $f(x)$ of bounded variation in $[a, b]$ and two additional numbers $\{f(a-0), f(b+0)\}$. To any $f \in V[a, b]$, a complex measure $d f$ corresponds in such a way that

$$
\left\{\begin{array}{l}
d f=\text { the usual one in }(a, b), \\
d f(a)=f(a+0)-f(a-0), \quad d f(b)=f(b+0)-f(b-0) .
\end{array}\right.
$$

We remark here that it is possible to define $g(x)=\int_{[a, x]} f(y) d Q(y) \in$ $V[a, b]$ for any $Q \in V[a, b]$ and $f \in L^{1}(|d Q|,[a, b])$ if we put $g(a-0)$ $=0$ and $g(b+0)=\int_{[a, b]} f(y) d Q(y)$.

Let $M$ and $Q \in V[a, b]$. Throughout this section we assume that $d M$ defines a nonnegative measure and $d Q$ defines a real one. We denote the right (left) derivative of a function $f$ at $x$ by $f^{+}(x)$ (resp. $f^{-}(x)$ ). Put

$D[a, b]=\left\{f \in C[a, b] ;\right.$ there exists an $f^{\perp} \in V[a, b]$ coinciding with $f^{+}(x)$ for every $x \in[a, b)$ and the measure $d f^{+}-f d Q$ is absolutely continuous with respect to $d M$ and its density belongs to $L^{2}(d M$, $[a, b])$.

$D_{\alpha, \beta}[a, b]=\left\{f \in D[a, b] ; f(a) \cos \alpha+f^{+}(a-0) \sin \alpha=0, \quad f(b) \cos \beta\right.$ $\left.+f^{+}(b+0) \sin \beta=0.\right\}$.

It is not difficult to see that any element of $D[a, b]$ is absolutely continuous with respect to the Lebesgue measure in $[a, b]$ and has the left derivative at each point in $(a, b]$. Let $L$ denote an operator defined by

$$
L f=-\frac{d f^{+}-f d Q}{d M}
$$

for $f \in D[a, b]$. Let $\varphi_{\alpha}(x, \lambda)$ and $\psi_{\beta}(x, \lambda)$ be solutions of the following 
integral equations

$$
\begin{aligned}
\varphi_{\alpha}(x, \lambda)= & -\sin \alpha+(x-a) \cos \alpha+\int_{[a, x]}(x-y) \varphi_{\alpha}(y, \lambda) d Q(y) \\
& -\lambda \int_{[a, x]}(x-y) \varphi_{\alpha}(y, \lambda) d M(y), \\
\psi_{\beta}(x, \lambda)= & -\sin \beta+(x-b) \cos \beta+\int_{[x, b]}(y-x) \psi_{\beta}(y, \lambda) d Q(y) \\
& -\lambda \int_{[x, b]}(y-x) \psi_{\beta}(y, \lambda) d M(y) .
\end{aligned}
$$

It is easy to see that these functions may be determined as the unique solutions of the following equations respectively

$$
\left\{\begin{array}{l}
L_{\varphi}=\lambda \varphi, \quad \varphi(a)=-\sin \alpha, \quad \varphi^{+}(a-0)=\cos \alpha \\
L \psi=\lambda \psi, \quad \phi(b)=-\sin \beta, \quad \phi^{+}(b+0)=\cos \beta .
\end{array}\right.
$$

Here we note the well-known identity between those functions of $D[a, b]$.

Lemma 1. 1. Let $f, g \in D[a, b]$ and let $c, d \in[a, b]$. Then we have

$$
\begin{aligned}
\int_{[\subset \pm 0, d \pm 0]}\{L f(x) & g(x)-f(x) \operatorname{Lg}(x)\} d M(x) \\
= & {\left[f(x) g^{+}(x)-f^{+}(x) g(x)\right]_{c \pm 0}^{d \pm 0}, }
\end{aligned}
$$

where $[c+0, d+0]=(c, d],[c-0, d-0]=[c, d)$ and so on.

The following comparison relation between zeros of solutions will be very usefull.

Lemma 1. 2. Let $\varphi, \psi$ be nontrivial solutions of $L \varphi=\lambda \varphi, L \psi=\mu \psi$ in $\left(x_{1}, x_{2}\right)$. Suppose $\psi\left(x_{1}\right)=\psi\left(x_{2}\right)=0$ and $\psi$ has no zeros in $\left(x_{1}, x_{2}\right)$. If $\lambda \geqq \mu$, then either $\varphi$ has at least one zero in $\left(x_{1}, x_{2}\right)$ or $\varphi$ is a constant multiple of $\psi$ in $\left(x_{1}, x_{2}\right)$. Here the latter case occurs only if $\lambda=\mu$ or $d M=0$ in $\left(x_{1}, x_{2}\right)$.

Proof. In Lemma 1. 1, substituting $c=x_{1}, d=x_{2}, f=\varphi$ and $g=\psi$, we have

$$
(\lambda-\mu) \int_{\left(x_{1}, x_{2}\right)} \varphi(x) \psi(x) d M(x)=\varphi\left(x_{2}\right) \psi^{+}\left(x_{2}-0\right)-\varphi\left(x_{1}\right) \psi^{+}\left(x_{1}+0\right),
$$


where we have used the assumption $\psi\left(x_{1}\right)=\psi\left(x_{2}\right)=0$. Since $\psi$ has no zeros in $\left(x_{1}, x_{2}\right)$, we may assume $\psi(x)>0$ for any $x \in\left(x_{1}, x_{2}\right)$. Then we have

$$
\psi^{+}\left(x_{2}-0\right)<0 \text { and } \psi^{+}\left(x_{1}+0\right)>0 .
$$

For at any fixed point $x$, it is impossible that $\psi(x)$ and $\psi^{ \pm}(x)$ vanish simultaneously.

Now we consider the two cases separately.

$\left.1^{\circ}\right)$ Either $\lambda=\mu$ or $d M=0$ in $\left(x_{1}, x_{2}\right)$. Then from $(1 \cdot 2)$ it follows that $\varphi\left(x_{2}\right) \psi^{+}\left(x_{2}-0\right)=\varphi\left(x_{1}\right) \psi^{+}\left(x_{1}+0\right)$. Noting (1.3), we see that either $\varphi\left(x_{1}\right)=\varphi\left(x_{2}\right)=0$ or $\varphi\left(x_{1}\right) \varphi\left(x_{2}\right)<0$ holds. Since under the condition $\left.1^{\circ}\right) \varphi$ and $\psi$ satisfy the same equation, the first case implies that $\varphi$ is a constant multiple of $\psi$. In the second case, from the continuity of $\varphi$ we see that there exists at least one zero of $\varphi$.

$\left.2^{\circ}\right) \quad \lambda>\mu$ and $d M=0$ in $\left(x_{1}, x_{2}\right)$. Suppose $\varphi$ has no zeros in $\left(x_{1}, x_{2}\right)$, hence assume $\varphi(x)>0$ for any $x \in\left(x_{1}, x_{2}\right)$. Then from $(1 \cdot 2)$ we have

$$
\varphi\left(x_{2}\right) \psi^{+}\left(x_{2}-0\right)>\varphi\left(x_{1}\right) \psi^{+}\left(x_{1}+0\right) \geqq 0,
$$

which implies $\varphi\left(x_{2}\right)<0$. This contradicts the assumption $\varphi(x)>0$ in $\left(x_{1}\right.$, $x_{2}$ ). This proves the lemma.

Since $\varphi_{\alpha}(x, \lambda)$ is a solution of $L \varphi=\lambda \varphi$, the set of zeros of $\varphi_{\alpha}(x, \lambda)$ in $[a, b]$ has no accumulating points. Let $\tau_{n}(\alpha, \lambda)$ be the $n$-th zero from the left end point $a$ of $[a, b]$, where $n=1,2, \cdots$. We denote the support of $d M$ by $F_{M}$ and put

$$
a_{0}=\inf F_{M}, b_{0}=\sup F_{M I} .
$$

Let $\varphi_{\alpha}(x)$ be $\varphi_{\alpha}(x, 0)$ and let $\left\{x_{1}, \cdots x_{n_{0}}\right\}$ be the set of zeros of $\varphi_{\alpha}(x)$ in $\left[a, a_{0}\right]$. Then it is obvious that every solution $\varphi_{\alpha}(x, \lambda)$ has the common zeros $\left\{x_{1}, x_{2}, \cdots x_{n_{0}}\right\}$.

\section{Proposition 1.3.}

(1) If $\lambda>\mu$, then $\tau_{n}(\alpha, \lambda) \leqq \tau_{n}(\alpha, \mu)$ for $n=1,2, \cdots$. Moreover $\tau_{n+n_{0}}(\alpha$, $\lambda)=\tau_{n+n_{0}}(\alpha, \mu)$ holds for some $n \geqq 1$ if and only if there exists a sequence $\left\{a_{k}\right\}_{k=1}^{n}$ such that 


$$
\left\{\begin{array}{l}
a_{k}=\tau_{k+n_{0}}(\alpha, \lambda) \quad \text { for } \quad \boldsymbol{R}^{1}, k=1,2, \cdots n, \\
F_{M} \cap\left[a, a_{n}\right] \subset\left\{a_{0}, a_{1}, a_{2}, \cdots a_{n}\right\} .
\end{array}\right.
$$

and theer exists a nontrivial solution $\varphi_{\alpha}$ of the equation

$$
\left\{\begin{array}{l}
d \varphi^{+}=\varphi d Q \quad \text { in } \quad\left[a, a_{n}\right] \\
\varphi(a) \cos \alpha+\varphi^{+}(a-0) \sin \alpha=0 \\
\varphi\left(a_{k}\right)=0 \text { for } k=0,1,2, \cdots n .
\end{array}\right.
$$

(2) $\tau_{n}(\alpha, \lambda)$ is continuous in $\lambda$ for every fixed $\alpha$.

Proof. First we consider the case $n=1$. Let $\xi=\tau_{n_{0}+1}(\alpha, \lambda)$ and $\eta=\tau_{n_{0}+1}(\alpha, \mu)$. Suppose $\xi>\eta$. In Lemma 1.1, substituting $c=a, d=\eta$, $f(x)=\varphi_{\alpha}(x, \lambda)$ and $g(x)=\varphi_{\alpha}(x, \mu)$, we have

$$
(\lambda-\mu) \int_{\left[a_{0}, \eta\right)} \varphi_{\alpha}(x, \lambda) \varphi_{\alpha}(x, \iota) d M I(x)=\varphi_{\alpha}(\eta, \lambda) \varphi_{\alpha}{ }^{+}(\eta-0, \mu),
$$

here we have used the facts that $\varphi_{\alpha}(a, \lambda)=\varphi_{\alpha}(a, \mu)=-\sin \alpha \cdot \varphi_{\alpha}{ }^{+}(a-0, \lambda)$ $=\varphi_{\alpha}{ }^{+}(a-0, \mu)=\cos \alpha, \varphi_{\alpha}(\eta, \mu)=0$ and $d M=0$ in $\left[a, a_{0}\right)$. Once we have obtained the identity $(1 \cdot 6)$, the situation becomes quite similar to the one of Lemma 1.2, so we stop going into details.

Next we consider the case $n>1$. It follows from Lemma 1.1 that $\tau_{n_{0}+n}(\alpha, \lambda) \leqq \tau_{n_{0}+n}(\alpha, \mu) . \quad$ Suppose $\quad \tau_{n_{0}+n}(\alpha, \lambda)=\tau_{\left.n_{0}\right\lrcorner n}(\alpha, \mu)$. Put $a_{k}=$ $\tau_{n_{0}+k}(\alpha, \mu)$ for $k=1,2, \cdots n$. Unless $\varphi_{\alpha}(x, \lambda)$ is a constant multiple of $\varphi_{\alpha}(x, \mu)$ in $\left(a_{n-1}, a_{n}\right), \tau_{n_{0}+n}(\alpha, \lambda)<\tau_{n_{0}+n}(\alpha, \mu)$. Hence it is necessary that $\varphi_{\alpha}(x, \lambda)$ and $\varphi_{\alpha}(x, \mu)$ are lineary dependent in $\left(a_{n-1}, a_{n}\right)$ and $d M=0$ in $\left(a_{n-1}, a_{n}\right)$. Consequently we have $\tau_{n_{0}+n-1}(\alpha, \lambda)=\tau_{n_{0}+n-1}(\alpha, \mu)$. Continuing this argument until $n=1$, we may prove (1).

Now let us prove (2). If $\alpha \equiv 0(\bmod \pi)$, then $\tau_{1}(\alpha, \lambda)=a$ for every $\lambda$, hence the continuity is trivial. So we may suppose $\alpha \neq 0(\bmod \pi)$. First we consider the case when

$$
a<\tau_{1}(\alpha, \lambda)<\tau_{2}(\alpha, \lambda)<\cdots<\tau_{n}(\alpha, \lambda)<b .
$$

For brevity we put $\varphi_{\lambda}(x)=\varphi_{\alpha}(x, \lambda)$ and $\tau_{k}(\lambda)=\tau_{k}(\alpha, \lambda) . \varphi_{\lambda}{ }^{+}(x)$ is continuous at each point $\tau_{k}(\lambda)$. This is because we have

$$
\varphi_{\lambda}{ }^{+}\left(\tau_{k}(\lambda)\right)-\varphi_{\lambda}{ }^{-}\left(\tau_{k}(\lambda)\right)=\varphi_{\lambda}\left(\tau_{k}(\lambda)\right)\left\{d Q\left(\tau_{k}(\lambda)\right)-\lambda d M\left(\tau_{k}(\lambda)\right)\right\}=0 .
$$

Therefore for any fixed sufficiently small $\varepsilon>0, \varphi_{\lambda}$ in $\left[\tau_{k}(\lambda)-\varepsilon, \tau_{k}(\lambda)\right)$ 
and $\varphi_{\lambda}$ in $\left(\tau_{k}(\lambda), \tau_{k}(\lambda)+\varepsilon\right]$ have different signs. Since $\varphi_{\mu}(x)$ converges to $\varphi_{\lambda}(x)$ uniformly in $[a, b]$ as $\mu \rightarrow \lambda$, there exists $\delta>0$ such that for any $\mu$ satisfying $|\mu-\lambda|<\delta, \varphi_{\mu}\left(x_{k}\right) \varphi_{\mu}\left(y_{k}\right)<0$ holds for some $x_{k} \in\left[\tau_{k}(\lambda)-\varepsilon\right.$, $\left.\tau_{k}(\lambda)\right)$ and $y_{k} \in\left(\tau_{k}(\lambda), \tau_{k}(\lambda)+\varepsilon\right]$. Hence $\varphi_{\mu}$ has at least one zero in $\left[\tau_{k}(\lambda)-\varepsilon, \tau_{k}(\lambda)+\varepsilon\right]$ for every $\mu,|\mu-\lambda|<\delta$. Again from the continuity of $\varphi_{\lambda}{ }^{+}(x)$ at $\tau_{k}(\lambda)$, we may assume that $\varphi_{\lambda}{ }^{+}(x)$ does not vanish in $\left[\tau_{k}(\lambda)-\varepsilon\right.$, $\left.\tau_{k}(\lambda)+\varepsilon\right]$. On the other hand, it is easy to see that $\varphi_{\mu}{ }^{+}\left(\varphi_{\mu}{ }^{-}\right)$also converges uniformly to $\varphi_{\lambda}{ }^{+}$(resp. $\left.\varphi_{\lambda}{ }^{-}\right)$. Hence $\varphi_{\mu}{ }^{ \pm}(x)$ have the same sign as $\varphi_{\lambda}{ }^{+}(x)$ for every $x \in\left[\tau_{k}(\lambda)-\varepsilon, \tau_{k}(\lambda)+\varepsilon\right]$ for $k=1,2, \cdots n$. Hence $\mid \tau_{k}(\lambda)$ $-\tau_{k}(\mu) \mid<\varepsilon$ for every $\mu$ such that $|\lambda-\mu|<\delta$.

In case $\tau_{n}(\lambda)=b$, we have only to extend the measures $d Q$ and $d M$ to a closed interval including $[a, b]$ in which $\varphi_{\alpha}(x, \lambda)$ has more than $n+1$ zeros. Then the problem may be reduced to the above case. This completes the proof of (2).

Since $\varphi_{\alpha}(x, \lambda)$ and $\varphi_{\alpha}{ }^{+}(x, \lambda)$ do not vanish simultaneously, we may define

$$
z_{\alpha}(x)=-\frac{\varphi_{\alpha}^{+}(x, \lambda)}{\varphi_{\alpha}(x, \lambda)}
$$

as a function taking the values in $\boldsymbol{R}^{1 \cup} \infty$.

Lemma 1.4. As far as $\varphi_{\alpha}(x, \lambda)$ dose not vanish, we have

$$
\left\{\begin{array}{l}
d z_{\alpha}(x)=z_{\alpha}(x)^{2} d x-d Q(x)+\lambda d M(x) \\
z_{\alpha}(a-0)=\cot \alpha
\end{array}\right.
$$

(2) $z_{\alpha}(x)$ is continuous at $\tau_{k}(\alpha, \lambda)$ and

$$
z_{\alpha}\left(\tau_{k}(\alpha, \lambda)-0\right)=+\infty, \quad z_{\alpha}\left(\tau_{k}(\alpha, \lambda)+0\right)=-\infty
$$

Proof. (1) may be proved by easy calculations. As for (2), we note that $\varphi_{\alpha}{ }^{+}(x, \lambda)$ is continuous at the zeros of $\varphi_{\alpha}(x, \lambda)$, which was proved in the argument in Proposition 1.3. Hence $z_{\alpha}(x)$ is continuous at $\tau_{k}(\alpha, \lambda)$. The last equalities are obvious, so we omit the proof. 
Proposition 1.5.

(1) If $0 \leqq \alpha<\beta<\pi$, then $\tau_{n}(\alpha, \lambda)<\tau_{n}(\beta, \lambda)<\tau_{n+1}(\alpha, \lambda)$.

(2) $\tau_{n}(\alpha, \lambda)$ is continuous in $\alpha \in[0, \pi)$.

(3) $\tau_{n}(\pi-0, \lambda)=\tau_{n+1}(0, \lambda)$ for $n=1,2, \cdots$.

Proof. We restrict $\alpha$ and $\beta$ to $[0, \pi)$ in the following argument and for brevity denote $\tau_{n}(\alpha)=\tau_{n}(\alpha, \lambda)$. First it should be noted that in any subinterval of $[a, b], \varphi_{\alpha}(x, \lambda)$ and $\varphi_{\beta}(x, \lambda)$ are linearly independent if $\alpha \neq \beta$. This is because every solution of $L \varphi=\lambda \varphi$ is uniquely determined by the values $\left\{\varphi(c), \varphi^{ \pm}(c)\right\}$ at arbitrary fixed point $c \in[a, b]$. Hence from Lemma 1.2 , there exists at least one zero of $\varphi_{\alpha}(x, \lambda)$ between two successive zeros of $\varphi_{\beta}(x, \lambda)$ and vice versa. Hence in order to prove (1) it is sufficient to verify $\tau_{1}(\alpha)<\tau_{1}(\beta)$. If $\alpha=0$ and $0<\beta<\pi$, then $0=$ $\tau_{1}(\alpha)<\tau_{1}(\beta)$, which proves (1). Suppose $0<\alpha<\beta<\pi$ and put $\delta(x)=$ $z_{\alpha}(x)-z_{\beta}(x), \delta_{0}=\cot \alpha-\cot \beta$. Then from (1) of Lemma 1.4 we have

$$
\left\{\begin{array}{l}
d \delta(x)=\left\{z_{\alpha}(x)+z_{\beta}(x)\right\} \delta(x) d x \\
\delta(a-0)=\delta_{0} .
\end{array}\right.
$$

Hence we see

$$
\delta(x)=\delta_{0} \exp \left\{\int_{a}^{x}\left(z_{\alpha}(y)+z_{\beta}(y)\right) d y\right\}
$$

as long as both $\varphi_{\alpha}(x, \lambda)$ and $\varphi_{\beta}(x, \lambda)$ do not vanish. Since $\delta_{0}>0$, we have by $(1 \cdot 7)$

$$
z_{\alpha}(x)>z_{\beta}(x)
$$

Suppose $\tau_{1}(\alpha)>\tau_{1}(\beta)$. Then combining (2) of Lemma 1.4 and (1.8), we have

$$
+\infty>z_{\alpha}\left(\tau_{1}(\beta)-0\right) \geqq z_{\beta}\left(\tau_{1}(\beta)-0\right)=+\infty,
$$

which is a contradiction. Consequently, noting $\varphi_{\alpha}$ and $\varphi_{\beta}$ have no common zeros, we see $\tau_{1}(\alpha)<\tau_{1}(\beta)$. This proves (1).

Let us fix $\alpha \in[0, \pi)$. Suppose $\tau_{n}(\alpha)<\tau_{n}(\beta)$ for some $\beta \in(0, \pi)$. Because $\varphi_{\alpha}(x, \lambda)$ may have as many zeros as we need by extending the measures $d Q$ and $d M$ appropriately, this assumption becomes no restriction. From (1), we have $\tau_{n}(\alpha+0) \geqq \tau_{n}(\alpha)$. Since $\varphi_{\alpha}(x, \lambda)$ is continuous 
in $(x, \alpha)$, we have $\varphi_{\alpha}\left(\tau_{n}(\alpha+0), \lambda\right)=0$. Suppose $\tau_{n}(\alpha+0)>\tau_{n}(\alpha)$. Then there exists only one zero of $\varphi_{\beta}(x, \lambda)$ in $\left(\tau_{n}(\alpha), \tau_{n}(\alpha+0)\right)$, which is equal to $\tau_{n}(\beta)$. This contradicts the fact $\tau_{n}(\alpha+0)<\tau_{n}(\beta)$. Hence $\tau_{n}(\alpha+0)=\tau_{n}(\alpha)$. We may prove also $\tau_{n}(\alpha-0)=\tau_{n}(\alpha)$ for $\alpha>0$. This proves the continuity of $\tau_{n}(\alpha)$.

In the inequality

$$
\tau_{n}(\alpha)<\tau_{n}(\beta)<\tau_{n+1}(\alpha),
$$

for $0 \leqq \alpha<\beta<\pi$, letting $\beta \rightarrow \pi$ and $\alpha=0$, we have

$$
\tau_{n}(\alpha)<\tau_{n}(\pi-0) \leqq \tau_{n+1}(0)
$$

Since $\tau_{n}(\pi-0)$ is a zero of $\varphi_{0}(x, \lambda)$, we have necessarily $\tau_{n}(\pi-0)=$ $\tau_{n+1}(0)$. This completes the proof.

Definition 1. 6. The pair $\left(L, D_{\alpha, \beta}[a, b]\right)$ is said to have the $E_{0}$ property if $F_{M}$ consists of finite elements and the equation

$$
\left\{\begin{array}{l}
d \varphi^{+}=\varphi d Q \quad \text { in } \quad[a, b] \\
\varphi(a) \cos \alpha+\varphi^{+}(a-0) \sin \alpha=0 \\
\varphi(b) \cos \beta+\varphi^{+}(b+0) \sin \beta=0 \\
\varphi(x)=0 \text { for every } x \in F_{M}
\end{array}\right.
$$

has a nontrivial solution.

Let $A_{\alpha, \beta}(\lambda)$ be the Wronskian of $\varphi_{\alpha}(x, \lambda)$ and $\psi_{\beta}(x, \lambda)$, namely

$$
\begin{aligned}
\Delta_{\alpha, \beta}(\lambda) & =\psi_{\beta}{ }^{+}(a-0, \lambda) \sin \alpha+\psi_{\beta}(a, \lambda) \cos \alpha \\
& =-\varphi_{\alpha}(b, \lambda) \cos \beta-\varphi_{\alpha}{ }^{+}(b+0, \lambda) \sin \beta .
\end{aligned}
$$

Proposition 1. 7. ( $\left.L, D_{\alpha, \beta}[a, b]\right)$ has the $E_{0}$-property if and only if $\Delta_{a, \beta}=0$. Moreover this is equivalent to that $\varphi_{\alpha}(x, \lambda)=0$, a.e. $d M$ in $[a, b]$ and $d_{\alpha, \beta}(\lambda)=0$ for some (or every) $\lambda$.

Proof. In Lemma 1. 1, substituting $f(x)=\varphi_{\alpha}(x, \lambda), g(x)=\varphi_{\alpha}(x, \mu)$, $c=a$ and $d=b$, we have

$$
(\lambda-, \iota) \int_{[a, b]} \varphi_{\alpha}(x, \lambda) \varphi_{\alpha}(x, \mu) d M(x)=\varphi_{\alpha}(b, \lambda) \varphi_{\alpha}^{\top}(b+0, ،)
$$




$$
-\varphi_{\alpha}(b, \mu) \varphi_{\alpha}^{+}(b+0, \lambda) .
$$

Suppose $\Delta_{\alpha, \beta}(\lambda)=0$ identically. Then by the definition of $\Delta_{\alpha, \beta}$, we have

$$
\varphi_{\alpha}(b, \lambda) \cos \beta+\varphi_{\alpha}^{+}(b+0, \lambda) \sin \beta=0 .
$$

Combining $(1 \cdot 10)$ and $(1 \cdot 11)$, we have

$$
\int_{[a, b]} \varphi_{\alpha}(x, \lambda) \varphi_{\alpha}(x, \mu) d M(x)=0
$$

for every $\lambda$, $\mu$. Hence we see $\varphi_{\alpha}(x, \lambda)=0$, a.e. $d M$ in $[a, b]$ for every $\lambda$. In particular, putting $\varphi(x)=\varphi_{\alpha}(x, 0)$, we see that $\varphi$ satisfies (1.9). Since $\varphi$ is nontrivial, this is possible only when $F_{M}$ has no accumulating points in $[a, b]$. Hence $F_{M}$ should be a finite set.

Conversely assume $(1.9)$ has a nontrivial solution $\varphi$. Then for any $\lambda$, we have

$$
d \varphi^{+}=\varphi d Q-\lambda \varphi d M
$$

Noting $\varphi(x)$ and $\varphi_{\alpha}(x, \lambda)$ satisfy the same boundary condition at $a$, we see that there exists a constant $C$ depending on $\lambda$ such that $\varphi_{\alpha}(x, \lambda)$ $=C \varphi(x)$ holds for any $x \in[a, b]$. Then we have

$$
\begin{aligned}
\Delta_{\alpha, \beta}(\lambda) & =C\left(\varphi(b) \cos \beta+\varphi^{+}(b+0) \sin \beta\right) \\
& =0,
\end{aligned}
$$

which completes the proof.

For simplicity, we denote

$$
\varphi_{0}(x)=\varphi_{\alpha}(x, 0), \quad \psi_{0}(x)=\psi_{\beta}(x, 0) .
$$

Define

$$
V(x, y)=\frac{1}{\Delta_{\alpha, \beta}(0)}\left\{\varphi_{0}(x) \psi_{0}(y)-\varphi_{0}(y) \psi_{0}(x)\right\},
$$

except for the case $\Delta_{\alpha, \beta}(0) \neq 0$.

Lemma 1. 8. Suppose $\Delta_{\alpha, \beta}(0) \neq 0$. Then $\varphi_{\alpha}(x, \lambda)$ and $\psi_{\beta}(x, \lambda)$ satisfy the integral equations 


$$
\left\{\begin{array}{l}
\varphi_{\alpha}(x, \lambda)=\varphi_{0}(x)-\lambda \int_{[a, x)} V(x, y) \varphi_{a}(y, \lambda) d M(y) \\
\psi_{\beta}(x, \lambda)=\psi_{0}(x)+\lambda \int_{(x, b]} V(x, y) \psi_{\beta}(x, \lambda) d M(y) .
\end{array}\right.
$$

Moreover $\varphi_{a}(x, \lambda)$ and $\psi_{\beta}(x, \lambda)$ have the estimates

$$
\begin{aligned}
& \left|\varphi_{\alpha}(x, \lambda)\right| \leqq C \cosh \left(c(x-a) M_{+}(x)|\lambda|\right)^{1 / 2} \\
& \left|\psi_{\beta}(x, \lambda)\right| \leqq C \cosh \left(c(b-x) M_{-}(x)|\lambda|\right)^{1 / 2},
\end{aligned}
$$

where $M_{+}(x)=\int_{[a, x)} d M(y), M_{-}(x)=\int_{(x, b]} d M(y)$ and $C$ is a constant.

Proof. (1.12) is easy to verify, so we omit the proof. Noting

$$
\Delta_{\alpha, \beta}(0) V(x, y)=\int_{x}^{y}\left\{\varphi_{0}(x) \psi_{0}^{-}(u)-\psi_{0}(x) \varphi_{0}{ }^{+}(u)\right\} d u
$$

we have

$$
|V(x, y)| \leqq C|x-y|
$$

for every $x, y \in[a, b]$. Then referring to I. S. Kac-M. G. Krein [10], we may obtain the inequality $(1 \cdot 13)$.

Definition 1.9. The pair ( $\left.L, D_{\alpha, \beta}[a, b]\right)$ is said to have the $E_{1}$-property if $F_{M}$ consists of finite elements and the equation

$$
d \varphi^{+}=\varphi d Q \text { in }[a, b]
$$

$$
\left\{\begin{array}{l}
\left\{\varphi(a) \cos \alpha+\varphi^{+}(a-0) \sin \alpha\right\}\left\{\varphi(b) \cos \beta+\varphi^{+}(b+0) \sin \beta\right\}=0 \\
\left\{\varphi(a) \cos \alpha+\varphi^{+}(a-0) \sin \alpha\right\}+\left\{\varphi(b) \cos \beta+\varphi^{+}(b+0) \sin \beta\right\} \neq 0 \\
\varphi(x)=0, \text { for } \quad x \in F_{u}
\end{array}\right.
$$

has a solution.

Proposition 1. 10. The entire function $\Delta_{\alpha, \beta}$ has no zeros if and only if $\left(L, D_{\alpha, \beta}[a, b]\right)$ has the $E_{1}$-property. Moreover this is equivalent to that either $\varphi_{a}(x, \lambda)$ or $\psi_{\beta}(x, \lambda)$ is equal to zero, a.e. $d M$ in $[a, b]$ for some (or every) $\lambda$ and $\Delta_{\alpha, \beta}$ is nontrivial.

Proof. From (1.13), it follows that $\Delta_{\alpha, \beta}(\lambda)=-\varphi_{\alpha}(b, \lambda) \cos \beta-$ 
$\varphi_{\alpha}{ }^{+}(b+0, \lambda) \sin \beta$ is an entire function of order at most $1 / 2$. Hence according to the Hadamard factorization theorem, $\Delta_{\alpha, \beta}$ has no zeros if and only if $\Delta_{\alpha, \beta}$ is a nonzero constant $\delta$. Without loss of generality, we may assume $\sin \beta \neq 0$. Then we have

$$
\varphi_{\alpha}^{+}(b+0, \lambda)=-\delta \operatorname{cosec} \beta-\varphi_{\alpha}(b, \lambda) \cot \beta .
$$

Hence from the identity $(1 \cdot 10)$, we have

$$
(\lambda-\mu) \int_{[a, b]} \varphi_{\alpha}(x, \lambda) \varphi_{\alpha}(x, \mu) d M(x)=\delta \operatorname{cosec} \beta\left[\varphi_{\alpha}(b, \mu)-\varphi_{\alpha}(b, \lambda)\right]
$$

for every $\lambda$, $\mu$. Putting $\mu=\bar{\lambda}$, we have

$$
(\operatorname{Im} \lambda) \int_{[a, b]}\left|\varphi_{\alpha}(x, \lambda)\right|^{2} d M(x)=-\delta \operatorname{cosec} \beta \operatorname{Im} \varphi_{\alpha}(b, \lambda) .
$$

Hence we see that $\operatorname{Im} \varphi_{\alpha}(b, \lambda) \geqq 0$ or $\leqq 0$ in $\mathbb{C}_{+}$according as $\delta \operatorname{cosec} \beta<0$ or $>0$. Since $\varphi_{\alpha}(b, \lambda)$ is an entire function, $\varphi_{\alpha}(b, \lambda)=p \lambda+q$ for some real numbers $p, q$ (cf. B.Ja. Levin [11] p. 230). Consequently we have

$$
\int_{[a, b]} \varphi_{\alpha}(x, \lambda) \varphi_{\alpha}(x, \mu) d M(x)=-p \delta \operatorname{cosec} \beta,
$$

for every $\lambda, \mu$. From this identity, it follows that for every $\lambda$

$$
\varphi_{\alpha}(x, \lambda)=\varphi_{\alpha}(x, 0)=\varphi_{0}(x)
$$

for every $x \in F_{M}$ holds. It is obvious that by the same argument as above we have for $\psi_{\beta}$

$$
\psi_{\beta}(x, \lambda)=\psi_{\beta}(x, 0)=\psi_{0}(x)
$$

for every $x \in F_{M}$. Hence from (1.12) it follows that

$$
\begin{aligned}
& \varphi_{0}(x) \int_{[a, x]} \psi_{0}(y) \varphi_{0}(y) d M(y)=\psi_{0}(x) \int_{[a, x]} \varphi_{0}(y)^{2} d M(y) \\
& \varphi_{0}(x) \int_{[x, b]} \psi_{0}(y)^{2} d M(y)=\psi_{0}(x) \int_{[x, b]} \varphi_{0}(y) \psi_{0}(y) d M(y)
\end{aligned}
$$

hold for every $x \in F_{M}$.

First we show that $F_{M}$ is a finite set. Assume $F_{M}$ is infinite. Then $F_{M}$ has an accumulating point $x_{0}$. Without loss of generality we may assume that $x_{0}$ is a right accumulating point. Then taking the right derivatives in $(1 \cdot 15)$ and $(1 \cdot 16)$ we have 
$(1 \cdot 17) \varphi_{0}{ }^{+}\left(x_{0}\right) \int_{\left[a, x_{0}\right]} \psi_{0}(y) \varphi_{0}(y) d M(y)=\psi_{0}{ }^{+}\left(x_{0}\right) \int_{\left[a, x_{0}\right]} \varphi_{0}(y)^{2} d M(y)$

$(1 \cdot 18) \quad \varphi_{0}{ }^{+}\left(x_{0}\right) \int_{\left(x_{0}, b\right]} \psi_{0}(y)^{2} d M(y)=\psi_{0}{ }^{+}\left(x_{0}\right) \int_{\left(x_{0}, b\right]} \varphi_{0}(y) \psi_{0}(y) d M(y)$.

Noting $\Delta_{\alpha, \beta}(0)=\delta \neq 0$, we see that $\varphi_{0}\left(x_{0}\right)$ and $\psi_{0}\left(x_{0}\right)$ do not vanish simultaneously. Hence

$$
\int_{\left[a, x_{0}\right]} \varphi_{0}(y)^{2} d M(y)+\int_{\left(x_{0}, b\right]} \psi_{0}(y)^{2} d M(y) \neq 0 .
$$

We may assume for instance $\int_{\left[a, x_{0}\right]} \varphi_{0}(y)^{2} d M(y) \neq 0$. In this case, from $(1 \cdot 15)$ and $(1 \cdot 17)$, it follows that $\varphi_{0}$ and $\psi_{0}$ are linearly dependent, which contradicts $\Delta_{\alpha, \beta}(0) \neq 0$. In this way, we may prove that $F_{M}$ is a finite set.

Let $F_{M}=\left\{x_{1}, x_{2}, \cdots x_{n}\right\}$, where $a \leqq x_{1}<x_{2} \cdots<x_{n} \leqq b$. Put $\varphi_{0}\left(x_{k}\right)$ $=\alpha_{k}, \psi_{0}\left(x_{k}\right)=\beta_{k}$ and $d M\left(x_{k}\right)=m_{k}$. Then (1.15) and (1.16) turn to the equations

$$
\begin{aligned}
& \alpha_{k} \sum_{j=1}^{k} \alpha_{j} \beta_{j} m_{j}=\beta_{k} \sum_{j=1}^{k} \alpha_{j}^{2} m_{j} \\
& \alpha_{k} \sum_{j=k}^{n} \beta_{j}^{2} m_{j}=\beta_{k} \sum_{j=k}^{n} \alpha_{j} \beta_{j} m_{j} .
\end{aligned}
$$

Supposing $\alpha_{k}=0$ for some $k \geqq 1$, we have from (1.19)

$$
\beta_{k} \sum_{j=1}^{k} \alpha_{j}^{2} m_{j}=0
$$

Noting $\varphi_{0}(x)$ and $\psi_{0}(x)$ do not vanish simultaneously, we have

$$
\sum_{j=1}^{k} \alpha_{j}{ }^{2} m_{j}=0
$$

which implies $\alpha_{j}=0$ for every $1 \leqq j \leqq k$. The parallel argument is possible also for $\beta_{j}$, hence we may assume that there exists a finite subset (possibly empty) $S$ of $\{1,2, \cdots n\}$ such that

$$
\left\{\begin{array}{l}
\alpha_{j} \neq 0, \beta_{j} \neq 0 \quad \text { for every } j \in S \\
\alpha_{j}=0 \text { for every } j<n_{1} \\
\beta_{j}=0 \text { for every } j>n_{2},
\end{array}\right.
$$

where $n_{1}=\min S$ and $n_{2}=\max S$. In this case, the equations (1.19) and $(1 \cdot 20)$ are valid even if we change $n$ to $n_{2}$ and 1 to $n_{1}$. Then it is 
easy to prove inductively that

$$
\beta_{j} / \alpha_{j}=\gamma \text { for every } j \in S .
$$

However putting $k=n_{1}-1$ in $(1 \cdot 20)$, we obtain

$$
\alpha_{n_{1}-1} \sum_{j=n_{1}-1}^{n_{2}} \beta_{j}^{2} m_{j}=\beta_{n_{1}-1} \sum_{j=n_{1}-1}^{n_{2}} \alpha_{j} \beta_{j} m_{j} .
$$

Noting $\alpha_{n_{1}-1}=0$ and $\beta_{n_{1}-1} \neq 0$, we have

$$
\sum_{j=n_{1}}^{n_{2}} \alpha_{j} \beta_{j} m_{j}=0
$$

Substituting $\beta_{j}=\gamma \alpha_{j}$ into the above identity, we see

$$
\gamma \sum_{j=n_{1}}^{n_{2}} \alpha_{j}^{2} m_{j}=0
$$

However this is possible only when $S$ is empty, which implies $\alpha_{j}=0$, $\beta_{j} \neq 0$ for every $j=1,2, \cdots n$. Hence in this case $\varphi(x)=\varphi_{\alpha}(x, 0)$ satisfies the equation $(1 \cdot 14)$.

Next suppose $\alpha_{j} \neq 0$ and $\beta_{j} \neq 0$ for every $j=1,2, \cdots n$. Then as we have seen in the above discussions, from $(1 \cdot 19)$ and $(1 \cdot 20)$ there exists some constant $\gamma$ such that

$$
\beta_{j}=\gamma \alpha_{j} \text { for every } j=1,2, \cdots n .
$$

Put $\varphi(x)=\psi_{\beta}(x, 0)-\gamma \varphi_{\alpha}(x, 0)$. Since $\psi_{\beta}$ and $\varphi_{\alpha}$ are linearly independent, $\varphi$ does not vanish identically. By the definition of $\varphi, \varphi$ satisfies the equation

$$
d \varphi^{+}=\varphi d Q-\lambda \varphi d M
$$

However we have assumed $\Delta_{\alpha, \beta}(\lambda) \neq 0$, hence $\varphi_{\alpha}(x, \lambda)$ and $\psi_{\beta}(x, \lambda)$ are linearly independent solutions of $(1 \cdot 21)$. Therefore there exist some constants $p(\lambda)$ and $q(\lambda)$ such that

$$
\varphi(x)=p(\lambda) \psi_{\beta}(x, \lambda)-q(\lambda) \varphi_{\alpha}(x, \lambda)
$$

holds for every $x \in[a, b]$. As has been seen,

$$
0=\varphi\left(x_{k}\right)=p(\lambda) \beta_{k}-q(\lambda) \alpha_{k}=\{\gamma p(\lambda)-q(\lambda)\} \alpha_{k}
$$

holds for every $x_{k} \in F_{M}$. Since $\alpha_{k} \neq 0$, we have

$$
q(\lambda)=\gamma p(\lambda) \text {. }
$$


Hence $\varphi(x)=p(\lambda)\left\{\psi_{\beta}(x, \lambda)-\gamma \varphi_{\alpha}(x, \lambda)\right\}$. Taking $x_{0} \in[a, b]$ such that $\varphi\left(x_{0}\right) \neq 0$, we have

$$
p(\lambda)^{-1}=\varphi\left(x_{0}\right)^{-1}\left\{\psi_{\beta}\left(x_{0}, \lambda\right)-\gamma \varphi_{\alpha}\left(x_{0}, \lambda\right)\right\} .
$$

From (1.13) we see that the right hand side is an entire function of order at most $1 / 2$. Since $p(\lambda)$ has no zeros, $p(\lambda)$ should be a nonzero constant. Since $p(0)=1$, we see $p(\lambda)=1$ identically. Consequently we have

$$
\left\{\begin{array}{l}
\varphi(x)=\psi_{\beta}(x, \lambda)-\gamma \varphi_{a}(x, \lambda) \\
\varphi^{+}(x)=\psi_{\beta}{ }^{+}(x, \lambda)-\gamma \varphi_{\alpha}^{+}(x, \lambda)
\end{array}\right.
$$

for every $x \in[a, b]$ and $\lambda$. Putting $x=b+0$, we have

$$
\varphi_{\alpha}(b, \lambda)=C_{1}, \quad \varphi_{\alpha}{ }^{+}(b+0, \lambda)=C_{2},
$$

where $C_{1}$ and $C_{2}$ are constants independent of $\lambda$. Choosing $\beta^{\prime}$ such that $C_{1} \cos \beta^{\prime}+C_{2} \sin \beta^{\prime}=0$, we consider a boundary value problem

$$
\left\{\begin{array}{l}
f(a) \cos \alpha+f^{+}(a-0) \sin \alpha=0 \\
f(b) \cos \beta^{\prime}+f^{+}(b+0) \sin \beta^{\prime}=0 .
\end{array}\right.
$$

Then by the definition of $\beta^{\prime}$, we have $\mathcal{A}_{\alpha, \beta^{\prime}}(\lambda)=0$ for every $\lambda$. Hence applying Proposition 1.7 , we see $\varphi_{\alpha}(x, 0)=0$, a.e. $d M$ in $[a, b]$, which contradicts the assumption that $\varphi_{\alpha}(x, 0)$ has no zeros in $F_{M}$.

Conversely suppose $\left(L, D_{\alpha, \beta}[a, b]\right)$ has the $E_{1}$-property. We may assume a solution of $(1 \cdot 14)$ satisfies the equation

$$
\left\{\begin{array}{l}
\varphi(a)=-\sin \alpha, \quad \varphi^{+}(a-0)=\cos \alpha \\
\varphi(b) \cos \beta+\varphi^{+}(b-0) \sin \beta \neq 0 .
\end{array}\right.
$$

Since $d \varphi^{+}=\varphi d Q-\lambda \varphi d M$ for every $\lambda$, we have

$$
\varphi_{\alpha}(x, \lambda)=\varphi(x)
$$

for every $x \in[a, b]$ and $\lambda$. Hence we have

$$
\begin{aligned}
\lrcorner_{\alpha, \beta}(\lambda) & =-\cos \beta \varphi_{\alpha}(b, \lambda)-\sin \beta \varphi_{\alpha}{ }^{\top}(b+0, \lambda) \\
& =-\cos \beta \varphi(b)-\sin \beta \varphi^{+}(b+0),
\end{aligned}
$$

which proves the proposition.

Theorem 1.11. Suppose that $\Delta_{\alpha, \beta}(\lambda)$ is a nontrivial function pos- 
sessing at least one zero. Let $\left\{\lambda_{n}\right\}$ be the set of zeros of $A_{\alpha, \beta}$ and arrange them according to magnitude. Let $N_{n}$ be the number of zeros of $\varphi_{\alpha}\left(x, \lambda_{n}\right)$. Then we have

(1) There exists the minimum $\lambda_{0}$ of $\left\{\lambda_{n}\right\}$.

(2) $N_{n}=n+\#\left\{x \in[a, b] ; \varphi_{\alpha}\left(x, \lambda_{0}\right)=0\right\}$.

Proof. From (1) of Proposition 1.3 it follows that $N_{n}$ is a nondecreasing sequence. First we show that $N_{n}$ is strictly increasing. Suppose $N_{n}=N_{n+1}$ for some $n$. Then from (1) of Proposition 1.3 it follows that

$$
\tau_{k}\left(\alpha, \lambda_{n+1}\right) \leqq \tau_{k}\left(\alpha, \lambda_{n}\right), \quad k=1,2, \cdots N_{n} .
$$

However the similar argument as in Proposition 1.3 is possible also for $\psi_{\beta}$, hence we obtain

$$
\tau_{k}\left(\alpha, \lambda_{n+1}\right) \geqq \tau_{k}\left(\alpha, \lambda_{n}\right), \quad k=1,2, \cdots N_{n} .
$$

Consequently we have

$$
\tau_{k}\left(\alpha, \lambda_{n+1}\right)=\tau_{k}\left(\alpha, \lambda_{n}\right), \quad k=1,2, \cdots N_{n} .
$$

Applying (1) of Proposition 1.3, we see that $\left(L, D_{\alpha, \beta}[a, b]\right)$ has the $E_{0}$-property, which contradicts the assumption that $\Delta_{\alpha, \beta}$ is nontrivial. Hence $N_{n}$ should be strictly increasing.

Let us prove the equality

$$
N_{n+1}=N_{n}+1
$$

First we consider the case $\beta=0$. Then the boundary condition at $b$ becomes $\varphi(b)=0$. Let $\lambda_{n}$ be any fixed zero of $\Delta_{\alpha, \beta}(\lambda)$. We consider the two cases below:

$\left.1^{\circ}\right)$ For any $\lambda>\lambda_{n}$, the number of zeros of $\varphi_{\alpha}(x, \lambda)$ in $[a, b]$ remains unchanged. Then since we have shown that $N_{n}$ is strictly increasing, we see that there exists no greater zeros than $\lambda_{n}$.

$\left.2^{\circ}\right)$ For some $\lambda>\lambda_{n}$, the number of zeros of $\varphi_{\alpha}(x, \lambda)$ in $[a, b]$ increases. Let $Q$ and $M$ extend to the right hand side of $b$ so that $\varphi_{\alpha}(x, \lambda)$ may have as many zeros in $[a, c]$ for some $c>b$ as we need. Let $\tau_{k}(\lambda)$ be the $k$-th zero of $\varphi_{\alpha}(x, \lambda)$ in $[a, c]$. Suppose $\varphi_{\alpha}\left(x, \lambda_{n}\right)$ has $p$ zeros in $[a, b]$. Then we have obviously 


$$
\tau_{p+1}\left(\lambda_{n}\right)>\tau_{p}\left(\lambda_{n}\right)=b .
$$

On the other hand, from the assumption

$$
\tau_{p+1}(\lambda) \leqq \tau_{p}\left(\lambda_{n}\right)=b
$$

for some $\lambda>\lambda_{n}$ follows. Since we have proved that $\tau_{p+1}(\lambda)$ is continuous (see Proposition 1.3), there exists $\mu>\lambda_{n}$ such that

$$
\tau_{p+1}(\mu)=b \text {. }
$$

Noting $\varphi_{\alpha}(b, \mu)=0$, we see $\mu \geqq \lambda_{n+1}$. Remembering that $N_{n}$ is increasing, we have $\mu=\lambda_{n+1}$, which proves $(1 \cdot 22)$ in case $\beta=0$.

In case $\beta \not \equiv 0(\bmod \pi)$, we may reduce the problem to the case $\beta=0$, by extending $d M$ and $d Q$ to $\left[a, x_{1}\right]$ as follows:

$$
\begin{aligned}
& d M=0 \text { in }\left(b, x_{1}\right] \\
& d Q(x)=q_{0} \delta_{\left\{x_{0}\right\}}(d x),
\end{aligned}
$$

where $x_{1}>x_{0}>b, q_{0} \in \boldsymbol{R}^{1}$ and $\delta_{\left\{x_{0}\right\}}(d x)$ is the Dirac measure at $x_{0}$. Here three constants should be chosen to satisfy the identity

$$
\left\{1+\left(x_{1}-x_{0}\right) q_{0}\right\} \sin \beta=\left\{\left(x_{1}-b\right)+\left(x_{0}-x_{1}\right) q_{0}\right\} \cos \beta .
$$

This is because for $x>x_{0}, \varphi_{\alpha}(x, \lambda)$ satisfies the equation

$$
\begin{aligned}
\varphi_{\alpha}(x, \lambda)= & \varphi_{\alpha}(b, \lambda)+(x-b) \varphi_{\alpha}^{+}(b+0, \lambda) \\
& +\int_{(b, x]}(x-y) \varphi_{\alpha}(y, \lambda) d Q(y) .
\end{aligned}
$$

Consequently the equality $(1 \cdot 22)$ has been established. This completes the proof.

Corollary 1. 12. Under the same condition as Theorem 1.11, we have

$$
\begin{aligned}
\#\left\{n ; \lambda_{n} \leqq \lambda\right\}= & \varepsilon(\lambda)+\#\left\{x \in[a, b] ; \varphi_{\alpha}(x, \lambda)=0\right\} \\
& -\#\left\{x \in[a, b] ; \varphi_{\alpha}\left(x, \lambda_{0}\right)=0\right\},
\end{aligned}
$$

where $|\varepsilon(\lambda)| \leqq 2$.

It is complicated to count the number of zeros of $\varphi_{\alpha}\left(x, \lambda_{0}\right)$ explicitly. However, for instance in case $d M(x)=d x$ or $d Q(x) \geqq 0$, that number 
becomes zero in $\left(a_{0}, b_{0}\right)$.

\section{$\S 2 . \quad$ Ergodic Property of the Solution of a Ricatti Equation with}

\section{a Random Coefficient}

Let $\{Q(x) ; x \in[0, \infty)\}$ be a random process with stationary independent increments whose characteristic function $\psi(\xi)$ defined by

$$
E\left(e^{i \xi Q(x)}\right)=e^{x \psi(\xi)}
$$

may be expressed as follows:

$$
\psi(\xi)=\int_{-\infty}^{\infty}\left(e^{i \xi u}-1\right) n(d u),
$$

where $n(d u)$ is a measure on $\mathbb{R}^{1}$ such that

$$
\int_{-\infty}^{\infty} \min (1,|u|) n(d n)<\infty
$$

Under this condition, $Q(x)$ becomes a function of bounded variation in each finite interval with probability one. Hence we may define the operator $L$ by

$$
L \varphi=-\frac{d \frac{d \varphi}{d x}-\varphi d Q(x)}{d x} .
$$

Let $\varphi$ be the solution of the equation

$$
\left\{\begin{array}{l}
L \varphi=\lambda \varphi \\
\varphi(0)=-\sin \alpha, \quad \varphi^{-}(0)=\cos \alpha .
\end{array}\right.
$$

Put $\xi(x)=\varphi(x), \eta(x)=\varphi^{+}(x)$ and $\zeta(x)=(\xi(x), \eta(x))$. Then $\zeta(x)$ satisfies the following stochastic differential equation

$$
\left\{\begin{array}{l}
d \xi(x)=\eta(x) d x \\
d \eta(x)=-\lambda \xi(x) d x+\xi(x) d Q(x) .
\end{array}\right.
$$

Without loss of generality, we may assume that $Q(x)$ is continuous at the origin almost surely. Hence the initial value of $\zeta(x)$ is $\zeta(0)=(-\sin \alpha$, $\cos \alpha)$.

Now let $G$ be a continuous map from $\boldsymbol{R}^{2} \backslash\{0\}$ to $\mathbb{R}^{1 \cup \infty}$ defined by

$$
G(\zeta)=-\frac{\eta}{\xi}, \quad \text { for } \quad \zeta=(\xi, \eta)
$$


Since the equation $(2 \cdot 1)$ is linear, we have immediately the following

Lemma 2. 1. Let $\zeta\left(. x, \zeta_{i}\right)$ be the solution of $(2 \cdot 1)$ with an initial value $\zeta_{i}(i=1,2)$. Suppose $G\left(\zeta_{i}\right)=G\left(\zeta_{2}\right)$. Then $G\left(\zeta\left(x, \zeta_{1}\right)\right)=G(\zeta(x$, $\left.\zeta_{2}\right)$ ) for any $x \geqq 0$.

Let us define a process $\{\approx(x)\}$ by

$$
\approx(x)=G(\zeta(x)) \text {. }
$$

Since $\{\zeta(x)\}$ is a strong Markov process on $\mathbb{R}^{2} \backslash\{0\}$, from the above lemma it follows that $\{z(x)\}$ also becomes a strong Markov process on $\boldsymbol{R}^{1 \cup \infty}$. The generator $A$ of the process has the form

$$
\left\{\begin{array}{l}
A f(z)=\left(z^{2}+\lambda\right) \frac{d f}{d z}+\int_{-\omega}^{\omega}\{f(z-u)-f(z)\} n(d u) \quad \text { for } z \neq \infty \\
A f(\infty)=-\lim _{z \rightarrow \infty} \approx\{f(z)-f(\infty)\} .
\end{array}\right.
$$

Let us define a sequence of random times by

$$
\left\{\begin{array}{l}
\tau_{1}=\inf \{x>0 ; z(x)=\infty\} \\
\tau_{n}=\inf \left\{x>\tau_{n-1} ; \approx(x)=\infty\right\}
\end{array}\right.
$$

Since $z(x)$ is continuous at each $\tau_{n}$ by (2) of Lemma 1.4 , it is easy to see that every $\tau_{n}$ becomes a Markov time.

We prepare some lemmas for our theorem.

Lemma 2.2. Let $n$ be a measure on $\boldsymbol{R}^{1}$ such that $\int_{-\infty}^{\omega} \min (1,|u|)$ $n(d u)<\infty$. Then for every fixed $\lambda>0$, we have the estimate

$$
\int_{-\infty}^{\omega} n(d u)\left|\int_{z}^{z-u} \frac{d y}{y^{2}+\lambda}\right|=O\left\{|z|^{-3 / 2}+\int_{|u|>|z| 1 / 2} n(d u)\right\}
$$

as $|\approx| \rightarrow \infty$.

Proof. First we consider the case $z \rightarrow+\infty$. We divide the above integral into two parts.

$$
\int_{-\infty}^{0} n(d u) \int_{z}^{z-u} \frac{d y}{y^{2}+\lambda}=\int_{-\infty}^{-z^{1 / 2}} n(d u) \int_{z}^{z-u} \frac{d y}{y^{2}+\lambda}+\int_{-z^{1 / 2}}^{-1} n(d u) \int_{z}^{z-u} \frac{d y}{y^{2}+\lambda}
$$




$$
\begin{aligned}
& +\int_{-1}^{0} n(d u) \int_{z}^{z-u} \frac{d y}{y^{2}+\lambda} \\
& \leqq \int_{-\infty}^{-z^{1 / 2}} n(d u) \int_{z}^{\infty} \frac{d y}{y^{2}+\lambda}+\int_{-\infty}^{-1} n(d u) \int_{z}^{z+z^{1 / 2}} \frac{d y}{y^{2}+\lambda}+\frac{1}{z^{2}+\lambda} \int_{-1}^{0}|u| n(d u) \\
& =O\left\{z^{-3 / 2}+\int_{-\infty}^{-z^{1 / 2}} n(d u)\right\} \\
& \int_{0}^{\infty} n(d u) \int_{z-u}^{z} \frac{d y}{y^{2}+\lambda}=\int_{0}^{1} n(d u) \int_{z-u}^{z} \frac{d y}{y^{2}+\lambda}+\int_{1}^{z^{1 / 2}} n(d u) \int_{z-u}^{z} \frac{d y}{y^{2}+\lambda} \\
& =O\left\{z^{-3 / 2}+\int_{z^{1 / 2}}^{\infty} n(d u)\right\} .
\end{aligned}
$$

As for the case $z \rightarrow-\infty$, we have only to put $\breve{h}(d u)=n(-d u)$, then we have

$$
\int_{-\infty}^{\infty} n(d u) \int_{-z}^{-z-u} \frac{d y}{y^{2}+\lambda}=\int_{-\infty}^{\infty} \breve{n}(d u) \int_{z}^{z-u} \frac{d y}{y^{2}+\lambda}
$$

Consequently we may obtain the expected estimate.

Lemma 2. 3. Let $n$ be the same measure as in Lemma 2. 2. Let $C_{b}\left(\boldsymbol{R}^{1}\right)$ be the space of bounded continuous functions on $\boldsymbol{R}^{1}$ with the supremum norm. For $g \in C_{b}\left(\boldsymbol{R}^{1}\right)$, we define

$$
N g(z)=\int_{-\infty}^{\infty} n(d u) \int_{z}^{z-u} \frac{g(y) d y}{y^{2}+\lambda}
$$

Then for given $h \in C_{b}\left(\boldsymbol{R}^{1}\right)$, the following equation is uniquely solvable in $C_{b}\left(\boldsymbol{R}^{1}\right)$.

$$
g(z)+N g(z)=h(z)
$$

Proof. First we prove that $N$ is completely continuous in $C_{b}\left(\boldsymbol{R}^{1}\right)$. Let $B$ be the unit ball in $C_{b}\left(\boldsymbol{R}^{1}\right)$. Applying Lemma 2.2, we have the estimate

$$
|N g(z)| \leqq \int_{-\infty}^{\infty} n(d u)\left|\int_{z}^{z-u} \frac{d y}{y^{2}+\lambda}\right|=O\left\{|z|^{-3 / 2}+\int_{|u| \geqq|z| 1 / 2} n(d u)\right\}
$$

uniformly with respect to $g \in B$. Putting 


$$
\delta\left(z, z_{0} ; u\right)=\sup _{g \in B}\left|\int_{z}^{z-u} \frac{g(y)}{y^{2}+\lambda} d y-\int_{z_{0}}^{z_{0}-u} \frac{g(y)}{y^{2}+\lambda} d y\right|
$$

for $z, z_{0} \in \boldsymbol{R}^{1}$, we have the estimates

$$
\delta\left(z, z_{0} ; u\right) \leqq 2 \min \left\{|u| / \lambda, \int_{-\infty}^{\infty} \frac{d y}{y^{2}+\lambda}\right\}
$$

and

$$
\delta\left(z, z_{0} ; u\right) \leqq(2 / \lambda)\left|z-z_{0}\right|
$$

Therefore, owing to the dominated convergence theorem, we have

$$
\sup _{g \in \boldsymbol{B}}\left|N g(z)-N g\left(z_{0}\right)\right| \leqq \int_{-\infty}^{\infty} \delta\left(z, z_{0} ; u\right) n(d u) \rightarrow 0
$$

as $z \rightarrow z_{0}$. From $(2 \cdot 3)$ and $(2 \cdot 4)$ we may conclude that the image $N(B)$ is relatively compact in $C_{b}\left(\boldsymbol{R}^{1}\right)$, hence $N$ is completely continuous. In order to prove that the equation $(2 \cdot 2)$ is uniquely solvable, it is sufficient to show that $\operatorname{Ker}(I+N)=0$. For $g \in \operatorname{Ker}(I+N)$, put

$$
f(z)=-\int_{z}^{\infty} \frac{g(y)}{y^{2}+\lambda} d y .
$$

Then we see

$$
\begin{gathered}
\left(z^{2}+\lambda\right) \frac{d f}{d z}+\int_{-\infty}^{\infty}\{f(z-u)-f(z)\} n(d u)=g(z)+\int_{-\infty}^{\infty} n(d u) \int_{z}^{z-u} \frac{g(y)}{y^{2}+\lambda} d y \\
=(I+N) g(z)=0 .
\end{gathered}
$$

Here we make use of the Markov process $\{z(x)\}$. The Dynkin formula gives us the identity

$$
E_{z}\left(f\left(z\left(T_{k}\right)\right)\right)-f(z)=E_{z}\left(\int_{0}^{T_{k}}(A f)(z(x)) d x\right)
$$

for any $z \neq \infty$, where $T_{k}=\min \left(k, \tau_{1}\right)$. As has been verified in the above argument, $A f(z)=0$, for any $z \neq \infty$. Hence we have

$$
f(z)=E_{z}\left(f\left(z\left(T_{k}\right)\right)\right) .
$$

Remembering (2) of Lemma 1.4, we see by letting $k$ to $+\infty$

$$
f(z)=E_{z}\left(f\left(z\left(\tau_{1}-0\right)\right)\right)=E_{z}(f(+\infty))=0 .
$$

This implies $\operatorname{Ker}(I+N)=0$, which completes the proof. 
Lemma 2. 4. For any $z \in \boldsymbol{R}^{1 \cup} \infty$ and $\lambda>0, E_{z}\left(\tau_{1}\right)$ is finite. $f(z)=E_{z}\left(\tau_{1}\right)$ is a unique solution of the equation

$$
\left\{\begin{array}{c}
\left(z^{2}+\lambda\right) \frac{d f}{d z}+\int_{-\infty}^{\infty}\{f(z-u)-f(z)\} n(d u)=-1 \\
f(+\infty)=\lim _{z \rightarrow+\infty} f(z)=0, \quad|f(-\infty)|<\infty .
\end{array}\right.
$$

Proof. Let us consider the integral equation

$$
g(z)+\int_{-\infty}^{\infty} n(d u) \int_{z}^{z-u} \frac{g(y)}{y^{2}+\lambda} d y=-1
$$

Applying Lemma 2.3, we have a unique solution $g(z)$ in $C_{b}\left(\mathbb{R}^{1}\right)$. Put

$$
f(z)=-\int_{z}^{\infty} \frac{g(y)}{y^{2}+\lambda} d y .
$$

Then $f$ satisfies the equation

$$
\left\{\begin{array}{c}
\left(z^{2}+\lambda\right) \frac{d f}{d z}+\int_{-\infty}^{\infty}\{f(z-u)-f(z)\} n(d u)=-1 \\
f(+\infty)=0, \quad|f(-\infty)|<\infty .
\end{array}\right.
$$

For $z \neq \infty$, the Dynkin formula leads us to

$$
E_{z}\left(f\left(z\left(T_{k}\right)\right)\right)-f(z)=E_{z}\left(\int_{0}^{T_{k}}(A f)(z(x)) d x\right) .
$$

Since, by the definition of $A, A f(z)=-1$ for any $z \neq \infty$, we have

$$
E_{z}\left(f\left(z\left(T_{k}\right)\right)\right)-f(z)=-E_{z}\left(T_{k}\right) .
$$

Letting $k$ to $+\infty$ and observing $z\left(\tau_{1}-0\right)=+\infty$, we have

$$
E_{z}\left(\tau_{1}\right)=f(z)-E_{z}(f(+\infty))=f(z)
$$

for any $z \neq \infty$. Applying Proposition 1.5, we have

$$
E_{\infty}\left(\tau_{1}\right)=\lim _{z \rightarrow-\infty} E_{z}\left(\tau_{1}\right)=f(-\infty) .
$$

This completes the proof.

Now we may prove the ergodic property of $\{z(x)\}$.

Theorem 2.5. For any fixed $\lambda>0,\{z(x)\}$ is ergodic, namely 
for any continuous function $\varphi$ in $\boldsymbol{R}^{1 \cup \infty}$, the equality

$$
\lim _{l \rightarrow \infty} \frac{1}{l} \int_{0}^{l} \varphi(z(x)) d x=\frac{1}{E_{\mathrm{\infty}}\left(\tau_{1}\right)} E_{m}\left(\int_{0}^{\tau_{1}} \varphi(z(x)) d x\right)
$$

holds almost surely for any $P_{z}$. Moreover the process has an invariant measure $T(z) d z$ rohich may be determined as a unique solution of the equation

$$
\left\{\begin{array}{c}
\frac{d}{d z}\left\{\left(z^{2}+\lambda\right) T(z)\right\}=\int_{-\infty}^{\infty}\{T(z+u)-T(z)\} n(d u) \\
\int_{-\infty}^{\infty} T(z) d z=1 .
\end{array}\right.
$$

Proof. Since we have observed in (2) of Lemma 1.4 that $z\left(\tau_{n}\right)=\infty$, from the strong Markov property of $\{z(x)\}$ it follows that the sequence of random variables

$$
\int_{\tau_{n}}^{\tau_{n-1}} \varphi(z(x)) d x, \quad n=1,2, \cdots
$$

is independent and has the same distribution as

$$
P_{m}\left\{\int_{0}^{\tau_{1}} \varphi(z(x)) d x<a\right\}
$$

with respect to any probability measure $P_{z}$. Since we have verified the finiteness of $E_{\infty}\left(\tau_{1}\right)$ in Lemma 2.4 , the strong law of large numbers gives us the identity

$$
\begin{aligned}
\lim _{l-\infty} \frac{1}{l} \int_{0}^{l} \varphi(z(x)) d x & =\lim _{n \rightarrow \infty} \frac{1}{\tau_{n}} \int_{n}^{\tau_{n}} \varphi(z(x)) d x \\
& =\lim _{n \rightarrow \infty} \frac{n}{\tau_{n}} \frac{1}{n} \sum_{k=1}^{n} \int_{\tau_{\tau_{1}}}^{\tau_{k}} \varphi(z(x)) d x \\
& =\frac{1}{E_{\infty}\left(\tau_{1}\right)} E_{m}\left(\int_{n}^{\tau_{1}} \varphi(z(x)) d x\right)
\end{aligned}
$$

almost surely for $P_{z}$, which proves the identity $(2 \cdot 6)$.

Let $g$ be a unique solution of the equation

$$
g(z)+\int_{-\infty}^{n} n(d u) \int_{z}^{7-u} \frac{g(y)}{y^{2}+\lambda} d y=-\varphi(z),
$$


whose existence was assured in Lemma 2. 3. Putting $h(z)=-\int_{z}^{\infty} \frac{g(y)}{y^{2}+\lambda} d y$, we have

$$
\left(z^{2}+\lambda\right) \frac{d h}{d z}+\int_{-\infty}^{\infty}\{h(z-u)-h(z)\} n(d u)=-\varphi(z) .
$$

As in the proof of Lemma 2.4, the Dynkin formula gives the identity

$$
h(z)=E_{z}\left(\int_{0}^{\tau_{1}} \varphi(z(x)) d x\right) .
$$

Here we define a function $S(z)$ by

$$
S(-z)=-\frac{d}{d z} E_{z}\left(\tau_{1}\right)
$$

then by $(2 \cdot 5) S$ satisfies the equation

$$
\left(z^{2}+\lambda\right) S(z)+\int_{-\infty}^{\infty} n(d u) \int_{z}^{z+u} S(y) d y=1 .
$$

The following formal calculation may be easily justified through the approximation of the measure $n$ by those with compact supports.

$$
\begin{aligned}
\int_{-\infty}^{\infty} \varphi(z) S(z) d z=-\int_{-\infty}^{\infty} & \left(z^{2}+\lambda\right) \frac{d h}{d z} S(z) d z \\
& -\int_{-\infty}^{\infty} S(z) d z \int_{-\infty}^{\infty}\{h(z-u)-h(z)\} n(d u) \\
= & h(-\infty)+\int_{-\infty}^{\infty} h(z) d z\left\{\frac{d}{d z}\left(z^{2}+\lambda\right) S(z)\right. \\
& \left.-\int_{-\infty}^{\infty}(S(z+u)-S(z)) n(d u)\right\},
\end{aligned}
$$

where we have used the fact $h(+\infty)=0$ and $\left(z^{2}+\lambda\right) S(z) \rightarrow 1$ as $|z| \rightarrow \infty$. The identity $(2 \cdot 8)$ implies that the second term vanishes identically, hence we have

$$
\int_{-\infty}^{\infty} \varphi(z) S(z) d z=h(-\infty)=E_{\infty}\left(\int_{0}^{\tau_{1}} \varphi(z(x)) d x\right)
$$

Then it is easy to see that $T(z)=\frac{S(z)}{E_{\infty}\left(\tau_{1}\right)}$ satisfies $(2 \cdot 7)$. Thus we obtain the theorem.

Here we define a spectral distribution function. Let $N(\lambda, I)$ be the 
number of eigenvalues not exceeding $\lambda$ for a certain boundary value problem of $L$ in the interval $I$.

Corollary 2. 6. (Rice formula) For any $\lambda>0$, we have the identity

$$
\lim _{l \rightarrow \infty} \frac{1}{l} N(\lambda,[0, l])=\lim _{|z| \rightarrow \infty} z^{2} T(z),
$$

almost surely for any $P_{z}$.

Proof. From Corollary 1.12, we have

$$
N(\lambda,[0, l])=\#\left\{n ; \tau_{n} \leqq l\right\}+\varepsilon(\lambda),
$$

where $|\varepsilon(\lambda)| \leqq 2$. Hence we obtain by the law of large numbers

$$
\begin{aligned}
\lim _{l \rightarrow \infty} \frac{1}{l} N(\lambda,[0, l]) & =\lim _{n \rightarrow m} n / \tau_{n} \\
& =1 / E_{\infty}\left(\tau_{1}\right) \text { a.s. } P_{z} .
\end{aligned}
$$

Noting $T(z)=S(z) / E_{\infty}\left(\tau_{1}\right)$ and $z^{2} S(z) \rightarrow 1$ as $|z| \rightarrow \infty$, we have easily the identity $(2 \cdot 9)$.

Definition 2. 7. We call the function $\lim _{l \rightarrow \infty} \frac{1}{l} N(\lambda,[0, l])$ the spectral distribution function of $L$ and denote it by $N(\lambda)$.

Corollary 2. 8. (Frisch-Lloyd [3]) Suppose

$$
\int_{|u|>1} \log |u| n(d u)<\infty .
$$

Then the function

$$
\varphi(s)=\int_{-\infty}^{\infty} e^{-i s z} T(z) d z
$$

satisfies the equations

$$
\left\{\begin{array}{c}
\frac{d^{2} \varphi}{d s^{2}}(s)=\left\{\lambda-\frac{\psi(s)}{i s}\right\} \varphi(s) \quad \text { in } \quad \boldsymbol{R}^{1} \backslash\{0\} \\
\varphi( \pm \infty)=0, \quad \varphi(0)=1,
\end{array}\right.
$$

and 


$$
N(\lambda)=-\frac{1}{\pi} \operatorname{Re} \frac{d \varphi}{d s}(0+),
$$

where

$$
\psi(s)=\int_{-\infty}^{\infty}\left(e^{i s u}-1\right) n(d u)
$$

Proof. Since $T(z)$ is integrable, we may take the Fourier transform of the both sides of $(2 \cdot 7)$ in the Schwartz distribution sense to obtain

$$
i s\left(-\frac{d^{2}}{d s^{2}} \varphi(s)+\lambda \varphi(s)\right)=\psi(s) \varphi(s) .
$$

The identities $\varphi( \pm \infty)=0$ and $\varphi(0)=1$ follow from the RiemannLebesgue theorem and the definition of $T(z)$ respectively, which proves $(2 \cdot 10)$. On the other hand, the assumption for $n(d u)$ implies

$$
\int_{|z| \geqq 1} \frac{d z}{|z|} \int_{|u| \geqq|r| 1 / 2} n(d u)<\infty
$$

Noting $|T(z)| \leqq \frac{C}{z^{2}+\lambda}$ for some constant $C$, we see by Lemma 2.2

$$
\int_{-\infty}^{\infty} \frac{|z| d z}{z^{2}+\lambda} \int_{-\infty}^{\infty} n(d u)\left|\int_{z}^{z+u} T(x) d x\right|<\infty
$$

By the definition of $N(\lambda)$ and $T(z)$, the equality

$$
T(z)=\frac{N(\lambda)}{z^{2}+\lambda}+\frac{1}{z^{2}+\lambda} \int_{-\infty}^{\infty} n(d u) \int_{z}^{z+u} T(x) d x
$$

holds. Applying the Fourier transform to the both sides, we have

$$
\varphi(s)=\frac{\pi}{\lambda^{1 / 2}} N(\lambda) e^{-\lambda 1 / 2|s|}+\int_{-\infty}^{\infty} \frac{e^{-i s z}}{z^{2}+\lambda} d z \int_{-\infty}^{\infty} n(d u) \int_{z}^{z+u} T(x) d x .
$$

Observing $(2 \cdot 12)$, we may differentiate the both sides to obtain

$$
\frac{d \varphi}{d s}=-\pi N(\lambda) e^{-\lambda^{1 / 2 s}}-i \int_{-\infty}^{\infty} \frac{z e^{-i s z}}{z^{2}+\lambda} d z \int_{-\infty}^{\infty} n(d u) \int_{z}^{z+u} T(x) d x .
$$

Since $T(x)$ is real, we have immediately

$$
\operatorname{Re} \frac{d \varphi}{d s}(0+)=-\pi N(\lambda)
$$

which completes the proof. 


\section{§ 3. Analytic Continuation of $\varphi(s)$ to the Upper Half Plane When the Support of $\boldsymbol{n}$ Is Contained in $(0, \infty)$}

We assume that the support of $n$ is contained in $(0, \infty)$ thereafter, whence the process $\{Q(x)\}$ is nondecreasing. Put

$$
V(s)=\frac{1}{i s} \int_{0}^{\infty}\left(e^{i u s}-1\right) n(d u)
$$

Then since $V(s)$ is holomorphic in the upper half plane $\mathbb{C}_{+}$, the FrischLloyd formula $(2 \cdot 10)$ may be studied on the pure imaginary axis. On this axis $V(s)$ is real valued and behaves like the function $s^{-\alpha}$, which makes it possible to apply the methods used in the scattering theory.

For this we need the following

Lemma 3. 1. Let $V(z)$ be a holomorphic function in $C_{+}$satisfying

(1) $\int_{1}^{\infty}\left|\frac{d V}{d z}\left(r e^{i \theta}\right)\right| d r<\infty$, for each $0<\theta<\pi$, and

(2) $V($ iy $)$ is real valued for every $y>0$,

(3) $\sup \{|V(z)| ;|z| \geqq M, \theta \leqq \arg z \leqq \pi-\theta\} \rightarrow 0$ as $M \rightarrow \infty$, for each $0<\theta$ $<\pi / 2$,

Then for each fixed $\lambda>0$, there exist unique linearly independent solutions $g_{ \pm}(z)$ of the equation

$$
\frac{d^{2}}{d z^{2}} g(z)=\{\lambda-V(z)\} g(z)
$$

such that

$$
\left\{\begin{array}{l}
g_{ \pm}(z) \sim \exp \left\{ \pm \int_{z_{0}}^{z}(\lambda-V(s))^{1 / 2} d s\right\} \\
\frac{d}{d z} g_{ \pm}(z) \sim \pm \lambda^{1 / 2} \exp \left\{ \pm \int_{z_{0}}^{z}(\lambda-V(s))^{1 / 2} d s\right\}
\end{array}\right.
$$

as $z \rightarrow \infty$ in each angle $\{z ; \theta \leqq$ arg $z \leqq \pi-\theta\}, 0<\theta<\pi / 2$, where we choose as a branch of $(\lambda-V(s))^{1 / 2}$ the one tending to $\lambda^{1 / 2}$ as $s \rightarrow \infty$, and $z_{0}$ is an arbitrary point in $C_{+}$such that $\lambda-V\left(z_{0}\right) \neq 0$.

Proof. Since it is not difficult to extend the way of R. Bellman 
[12] (ch. 2, Th. 8, p.50) to the above complex variable case, we omit the proof.

Q.E.D.

Since in $\mathbb{C}_{+}$we have

$$
V(z)=\int_{0}^{\infty} e^{i u z} d u \int_{u}^{\infty} n(d t)
$$

the inequality

$$
\int_{1}^{\infty}\left|\frac{d V}{d z}\left(r e^{i \theta}\right)\right| d r \leqq \frac{1}{\sin \theta} \int_{0}^{\infty} \exp (-u \sin \theta) d u \int_{u}^{\infty} n(d t)<\infty
$$

follows from $\int_{0}^{1} d u \int_{u}^{\infty} n(d t)<\infty$. Hence $V(z)$ satisfies the all conditions of Lemma 3.1. Put

$$
U(x)=V(i x)
$$

Theorem 3.2. Suppose

$$
\int_{1}^{\infty} \log u n(d u)<\infty
$$

Then for each $\lambda>0$, we have

$$
N(\lambda)=\frac{\lambda^{1 / 2}}{\pi\left|f_{-}(0, \lambda)\right|^{2}},
$$

where $f_{-}$is a unique solution of the equation

$$
\left\{\begin{aligned}
\frac{d^{2}}{d x^{2}} f_{-}(x) & =\{-\lambda+U(x)\} f_{-}(x) \\
f_{-}(x) & \sim \exp \left\{-i \int_{x_{0}}^{x}(\lambda-U(y))^{1 / 2} d y\right\}
\end{aligned}\right.
$$

as $x \rightarrow+\infty . \quad x_{0}$ is any positive number such that $\lambda-U\left(x_{0}\right) \neq 0$.

Proof. First we prove that the analytic continuation of $\varphi$, which will be denoted by the same notation, is linearly dependent on $g_{-}$of Lemma 3.1. Since $g_{ \pm}$are linearly independent solutions, there exist two constants $a, b$ such that

$$
\varphi(z)=a g_{+}(z)+b g_{-}(z) .
$$

Since $g_{ \pm}$are bounded on the upper pure imaginary axis, so is $\varphi$. More- 
over, by the definition of $\varphi, \varphi$ must be bounded also on $\boldsymbol{R}^{1}$. The estimate (3.1) implies that $\varphi$ is a holomorphic function in $\boldsymbol{C}_{+}$with exponential type at most $\lambda^{1 / 2}$. Hence according to the Phragmén-Lindelöf theorem, $\varphi$ is bounded in $\boldsymbol{C}_{+}$. On the other hand, noting that for each fixed $0<\theta<\pi / 2, g_{+}\left(r e^{i \theta}\right)$ is of exponential growth and $g_{-}\left(r e^{i \theta}\right)$ is of exponential decay, we may conclude that $a$ is zero, whence we have

$$
\varphi(z)=b g_{-}(z)
$$

$\varphi(z)$ may be irregular at the origin. However the assumption $\int_{1}^{\infty} \log u$ $n(d u)<\infty$ implies

$$
\int_{0+}\left|U\left(r e^{i \theta}\right)\right| d r<\infty
$$

for each $0 \leqq \theta \leqq \pi$, whence we may avoid that possibility. In particular, we have $\frac{d \varphi}{d s}(0+)=\frac{d \varphi}{d s}(i 0+)$, which together with $(2 \cdot 11)$ gives us the identity

$$
N(\lambda)=-\frac{1}{\pi} \operatorname{Re} \frac{d \varphi}{d s}(i 0+)
$$

Now put $f_{-}(x)=g_{-}(i x)$. Then from $(3 \cdot 4)$ and $\varphi(0)=1$, we have

$$
\varphi(z)=\frac{f_{-}(-i z)}{f_{-}(0)}, \quad \frac{d \varphi}{d z}=-i \frac{\frac{d}{d z} f_{-}(-i z)}{f_{-}(0)} .
$$

By the definition, $f_{-}$is a solution of the equation

$$
\left\{\begin{array}{l}
\frac{d^{2}}{d x^{2}} f_{-}(x)=\{-\lambda+U(x)\} f_{-}(x) \\
f_{-}(x) \sim \exp \left\{-i \int_{x_{0}}^{x}(\lambda-U(y))^{1 / 2} d y\right\} \\
\frac{d}{d x} f_{-}(x) \sim-i \lambda^{1 / 2} \exp \left\{-i \int_{x_{0}}^{x}(\lambda-U(y))^{1 / 2} d y\right\},
\end{array}\right.
$$

as $x \rightarrow+\infty$. Since $U(x)$ is real valued for each positive number $x$, the conjugate function $\bar{f}_{-}$of $f_{-}$is also a solution of $(3 \cdot 5)$ with the conjugate asymptotic behaviour of $f_{-}$. Hence according to the invariance of the Wronskian, we have

$$
\begin{aligned}
\frac{d}{d x} f_{-}(0+) \overline{f_{-}(0)}-f_{-}(0) \overline{\frac{d}{d x} f_{-}(0+)} & =\frac{d}{d x} f_{-}(x) \overline{f_{-}(x)}-f_{-}(x) \overline{\frac{d}{d x} f_{-}(x)} \\
& =-2 i \lambda^{1 / 2} .
\end{aligned}
$$


Consequently we have

$$
N(\lambda)=-\frac{1}{\pi} \operatorname{Im} \frac{\frac{d}{d x} f_{-}(0+)}{f_{-}(0)}=\frac{\lambda^{1 / 2}}{\pi\left|f_{-}(0)\right|^{2}},
$$

which completes the proof.

\section{$\S 4$. Asymptotic Behaviour of $N(\lambda)$ at the Origin}

In the previous section, our problem was reduced to the analysis of a familiar equation

$$
\frac{d^{2} f}{d x^{2}}=\{-\lambda+U(x)\} f(x)
$$

Observing $U(x)=\int_{0}^{\infty} e^{-x u} \int_{u}^{\infty} n(d t)$, we see that $U$ is strictly monotone decreasing and $U(+\infty)=0$. Let $x(\lambda)$ denote a unique solution of $U(x)$ $=\lambda$. As long as we are concerned with small $\lambda>0$, there are no problems whether the solution exists or not for large $\lambda$. Define $\zeta$ by

$$
\zeta(x)=\left\{\begin{array}{l}
\int_{x(\lambda)}^{x}(\lambda-U(y))^{1 / 2} d y, \quad \text { for } x \geqq x(\lambda), \\
e^{-3 \pi i / 2} \int_{x}^{x(\lambda)}(U(y)-\lambda)^{1 / 2} d y, \text { for } 0<x<x(\lambda) .
\end{array}\right.
$$

Put

$$
\left\{\begin{array}{l}
Q(x)=-\frac{1}{4} \frac{d^{2} U / d x^{2}}{(\lambda-U(x))^{2}}-\frac{5}{16} \frac{(d U / d x)^{2}}{(\lambda-U(x))^{3}}+\frac{5}{36} \frac{1}{\zeta(x)^{2}}, \\
\eta_{0}(\zeta)=(\pi \zeta)^{1 / 2} H_{\nu}^{(2)}(\zeta), \quad \eta_{1}(\zeta)=(\pi \zeta)^{1 / 2} H_{\nu}^{(1)}(\zeta),
\end{array}\right.
$$

where $\nu=1 / 3$ and $H_{\nu}{ }^{(i)}$ is the Hankel function. Define a Green function

$$
G(\zeta, \theta)=\frac{1}{4 i}\left\{\eta_{1}(\zeta) \eta_{0}(\theta)-\eta_{1}(\theta) \eta_{0}(\zeta)\right\}
$$

Then the Liuoville transformation of $(4 \cdot 1)$ by the variable $\zeta(x)$ leads us to the integral equation

$$
g(x)=\eta_{0}(\zeta(x))-\int_{x}^{\infty} G(\zeta(x), \zeta(y)) g(y) Q(y)(\lambda-U(y))^{1 / 2} d y,
$$

where $g(x)=(\lambda-U(x))^{1 / 4} f(x)$.

This procedure, which was found out by R.E. Langer, is stated in 
E. C. Titchmarsh [13] (p. 356 ) and very effective in studing the behaviour of the solution of $(4 \cdot 1)$ with respect to $(x, \lambda)$ simultaneously.

For the latter purpose, we give here the asymptotic behaviours of $\eta_{k}$

$$
\left\{\begin{array}{l}
\eta_{0}(\zeta) \sim 2^{1 / 2} \exp \left(i \zeta-\frac{2 \nu+1}{4} \pi i\right) \\
\eta_{1}(\zeta) \sim 2^{1 / 2} \exp \left(-i \zeta+\frac{2 \nu+1}{4} \pi i\right)
\end{array}\right.
$$

as $\zeta \rightarrow \infty . \quad G$ and $\frac{\partial G}{\partial \zeta}$ also have the estimates

$$
\left\{\begin{array}{l}
|G(\zeta, \theta)| \leqq C \\
\left|\frac{\partial G(\zeta, \theta)}{\partial \zeta}\right| \leqq C((1+\zeta) / \zeta)^{5 / 6}, \quad \text { for } \quad \zeta, \theta \geqq 0,
\end{array}\right.
$$

$(4 \cdot 5) \quad|G(\zeta(x), \zeta(y))| \leqq C \exp (|\zeta(x)|-|\zeta(y)|)$, for $0<x \leqq y \leqq x(\lambda)$,

where $C$ is a constant independent of both $\lambda$ and the variable $x$.

Here we give some lemmas for later use.

\section{Lemma 4. 1 .}

$$
\lim _{x \rightarrow \infty} x U(x)=-\lim _{x \rightarrow \infty} x^{2} \frac{d U}{d x}=\int_{0}^{\infty} n(d u) .
$$

(2) $\frac{d U}{d x} / U(x) \geqq \frac{d^{2} U}{d x^{2}} / \frac{d U}{d x} \geqq \cdots$, and each one is negative monotone increasing function.

$$
-\frac{d^{k+1} U}{d x^{k+1}} / \frac{d^{k} U}{d x^{k}} \leqq \frac{k+1}{x}, \text { for } k=0,1,2, \cdots
$$

Proof. From the identities

$$
\begin{aligned}
x U(x) & =\int_{0}^{\infty}\left(1-e^{-x u}\right) n(d u) \text { and }-x^{2} \frac{d U}{d x} \\
& =\int_{0}^{\infty}\left(1-e^{-x u}-x u e^{-x u}\right) n(d u),
\end{aligned}
$$

(1) follows immediately. (2) results from the Schwarz inequality.

may be proved by simple computations, whence we omit the proof. 
For further developments we must impose a restriction on $U(x)$, namely

$$
-x \frac{d U}{d x} / U(x) \geqq c>0
$$

holds for every sufficiently large $x$.

Lemma 4. 2. Under the condition (4.6), we have the estimates for every $a>1$ and $b<1$,

$$
\begin{gathered}
a^{-1 / c} x(\lambda) \leqq x(a \lambda) \leqq a^{-1} x(\lambda), \\
a^{-1} U(x) \leqq U(a x) \leqq a^{-c} U(x), \text { for every sufficiently small } \lambda
\end{gathered}
$$

and large $x$, and

$$
|\zeta(x(a \lambda))| \rightarrow \infty \text { and }|\zeta(x(b \lambda))| \rightarrow \infty \text { as } \lambda \rightarrow 0 \text {. }
$$

Proof. From Lemma 4.1, we have $-x \frac{d U}{d x} \leqq U(x)$. Putting $x=x(\lambda)$, we see

$$
-x(\lambda) \frac{d U}{d x}(x(\lambda)) \leqq U(x(\lambda))=\lambda .
$$

Further noting $\frac{d x}{d \lambda}=\frac{d U}{d x}(x(\lambda))^{-1}$, we have $-\frac{d x}{d \lambda} / x(\lambda) \geqq 1 / \lambda$. This implies the inequality

$$
\log (x(\lambda) / x(a \lambda))=-\int_{\lambda}^{a \lambda} \frac{d x}{d u} / x(u) d u \geqq \int_{\lambda}^{a \lambda} \frac{d u}{u}=\log a,
$$

which proves the inequality $(4 \cdot 7)$. We may show (4.8) similarly.

Since we have for $y<x(\lambda)$

$$
U(y)-\lambda=-\int_{y}^{x(\lambda)} \frac{d U}{d u} d u \geqq-\frac{d U}{d x}(x(\lambda))(x(\lambda)-y),
$$

whence noting $(4 \cdot 7)$ we obtain

$$
\begin{aligned}
|\zeta(x(a \lambda))| & =\int_{x(a \lambda)}^{x(\lambda)}(U(y)-\lambda)^{1 / 2} d y \geqq \frac{2}{3}\left(-\frac{d U}{d x}(x(\lambda))\right)^{1 / 2}(x(\lambda)-x(a \lambda))^{3 / 2} \\
& \geqq \frac{2}{3}\left(1-a^{-1}\right)^{3 / 2}\left(-x(\lambda)^{2} \frac{d U}{d x}(x(\lambda))\right)^{1 / 2} x(\lambda)^{1 / 2} .
\end{aligned}
$$

Since $x(\lambda) \rightarrow \infty$ as $\lambda \rightarrow 0$ and $-x(\lambda) \frac{{ }^{2}}{d U} \frac{d U}{d x}(x(\lambda))$ is bounded from below by 
some positive number as $\lambda \rightarrow 0$, we have $|\zeta(x(a \lambda))| \rightarrow \infty$ as $\lambda \rightarrow 0$. The second one may be proved similarly.

Lemma 4. 3. Under the condition (4.6) we obtain the estimates

$$
\begin{aligned}
& \int_{x(a \lambda)}^{\infty}\left|Q(y)(\lambda-U(y))^{1 / 2}\right| d y=o(1), \\
& \int_{x}^{x(a \lambda)}\left|Q(y)(\lambda-U(y))^{1 / 2}\right| d y=o(1)
\end{aligned}
$$

as $\lambda \rightarrow 0$ for every sufficiently small $a>1$.

Proof. Put

$$
\begin{cases}I_{1}=\int_{x(b \lambda)}^{\infty}\left|Q(y)(\lambda-U(y))^{1 / 2}\right| d y, \quad I_{2}=\int_{x(\lambda)}^{x(b \lambda)}\left|Q(y)(\lambda-U(y))^{1 / 2}\right| d y, \\ I_{3}=\int_{x(a \lambda)}^{x(\lambda)}\left|Q(y)(\lambda-U(y))^{1 / 2}\right| d y, & I_{4}=\int_{x}^{x(a \lambda)}\left|Q(y)(\lambda-U(y))^{1 / 2}\right| d y .\end{cases}
$$

Then we have

$$
\int_{x(a \lambda)}^{\infty}\left|Q(y)(\lambda-U(y))^{1 / 2}\right| d y=I_{1}+I_{2}+I_{3}
$$

Since we may treat $I_{1}$ and $I_{4}$ (resp. $I_{2}$ and $I_{3}$ ) analogously, we prove only the estimates for $I_{1}$ and $I_{2}$.

By the definition of $Q$, we have

$$
\begin{aligned}
I_{1} \leqq & \frac{1}{4} \int_{x(b \lambda)}^{\infty} \frac{d^{2} U / d x^{2}}{(\lambda-U(x))^{3 / 2}} d x+\frac{5}{16} \int_{x(b \lambda)}^{\infty} \frac{(d U / d x)^{2}}{(\lambda-U(x))^{5 / 2}} d x \\
& +\frac{5}{36} \int_{x(b \lambda)}^{\infty} \frac{(\lambda-U(x))^{1 / 2}}{\zeta(x)} d x
\end{aligned}
$$

which we denote by $J_{1}, J_{2}$ and $J_{3}$ respectively. Noting the inequality

$$
\lambda-U(x)=(1 / b) U(x(b \lambda))-U(x) \geqq(1 / b-1) U(x),
$$

in order to verify that $J_{1}$ and $J_{2}$ tend to zero as $\lambda \rightarrow 0$, we have only to show

$$
\int_{1}^{\infty}\left(\frac{d^{2} U / d x^{2}}{U(x)^{3 / 2}}+\frac{(d U / d x)^{2}}{U(x)^{5 / 2}}\right) d x<\infty .
$$

From Lemma 4. 1, it follows that 


$$
\begin{aligned}
\int_{1}^{\infty} \frac{d^{2} U / d x^{2}}{U(x)^{3 / 2}} d x & =\int_{1}^{\infty} \frac{d^{2} U / d x^{2}}{-d U / d x} \frac{1}{U(x)} \frac{-d U / d x}{U(x)^{1 / 2}} d x \\
& \leqq \int_{1}^{\infty} \frac{2}{x U(x)} \frac{-d U / d x}{U(x)^{1 / 2}} d x \leqq C U(1)^{1 / 2}<\infty
\end{aligned}
$$

and

$$
\begin{aligned}
\int_{1}^{\infty} \frac{(d U / d x)^{2}}{U(x)^{5 / 2}} d x & =\int_{1}^{\infty} \frac{-d U / d x}{U(x)} \frac{1}{U(x)} \frac{-d U / d x}{U(x)^{1 / 2}} d x \\
& \leqq C \int_{1}^{\infty} \frac{-d U / d x}{U(x)^{1 / 2}} d x \leqq 2 C U(1)^{1 / 2}<\infty
\end{aligned}
$$

On the other hand, the identity

$$
J_{3}=\frac{5}{36} \int_{x(b \lambda)}^{\infty} \frac{d \zeta / d x}{\zeta(x)} d x=\frac{5}{36} \frac{1}{\zeta(x(b \lambda))}
$$

and the estimate (4.9) imply $J_{3} \rightarrow 0$ as $\lambda \rightarrow 0$. This proves that $I_{1} \rightarrow 0$ as $\lambda \rightarrow 0$.

Put

$$
\delta(x)=\frac{2}{5} \frac{d U / d x}{(\lambda-U(x))^{3 / 2}} \int_{x(\lambda)}^{x} \frac{(\lambda-U(y))^{3 / 2} d^{2} U / d y^{2}}{(d U / d y)^{2}} d y
$$

Then $Q(x)$ may be expressed by $\delta(x)$ as follows:

$$
Q(x)=\frac{5}{16} \frac{(d U / d x)^{2}}{(\lambda-U(x))^{3}} \frac{\delta(x)^{2}(3+2 \delta(x))}{1+\delta(x)} .
$$

First note the inequality

$$
\begin{aligned}
|\delta(x)| & \leqq \frac{2}{5} \frac{-d U / d x}{(\lambda-U(x))^{3 / 2}}(\lambda-U(x))^{3 / 2} \int_{x(\lambda)}^{x} \frac{d^{2} U / d y^{2}}{(d U / d y)^{2}} d y \\
& =\frac{2}{5}-\frac{1}{-\frac{d U}{d x}(x(\lambda))}\left\{\frac{d U}{d x}(x)-\frac{d U}{d x}(x(\lambda))\right\} \\
& =\frac{2}{5} \frac{1}{-\frac{d U}{d x}(x(\lambda))} \int_{x(\lambda)}^{x} \frac{d^{2} U}{d y^{2}} d y \leqq \frac{2}{5} \frac{d^{2} U / d x^{2}}{-d U / d x}(x(\lambda))(x-x(\lambda)) \\
(4 \cdot 13) \quad & \leqq \frac{4}{5} \frac{x-x(\lambda)}{x(\lambda)} .
\end{aligned}
$$

This together with $(4 \cdot 7)$ gives us the inequality for $x(\lambda) \leqq x \leqq x(b \lambda)$ 


$$
|\delta(x)| \leqq \frac{4}{5}\left(b^{-1 / c}-1\right) .
$$

Choose $b<1$ so that $|\delta(x)| \leqq \frac{1}{2}$ may hold for every $x(\lambda) \leqq x \leqq x(b \lambda)$. Then the main term of $Q$ becomes

$$
Q_{0}(x)=\frac{25}{16} \frac{(d u / d x)^{2}}{(\lambda-U(x))^{3}} \delta(x)^{2} .
$$

Noting for $x \geqq x(\lambda)$

$$
\lambda-U(x)=-\int_{x(\lambda)}^{x} \frac{d U}{d y} d y \geqq-\frac{d U}{d x}(x-x(\lambda))
$$

and applying $(4 \cdot 13)$, we have

$$
\begin{aligned}
\int_{x(\lambda)}^{x(b \lambda)} Q_{0}(x)(\lambda-U(x))^{1 / 2} d x \\
\quad \leqq \frac{1}{x(\lambda)^{2}} \int_{x(\lambda)}^{x(b \lambda)} \frac{(d U / d x)^{2}}{(\lambda-U(x))^{3}}(\lambda-U(x))^{1 / 2}(x-x(\lambda))^{2} d x \\
\quad \leqq \frac{1}{x(\lambda)^{2}} \int_{x(\lambda)}^{x(b \lambda)}\left(-\frac{d U}{d x}(x-x(\lambda))\right)^{-1 / 2} d x \\
\quad \leqq x(\lambda)^{-2}\left(-\frac{d U}{d x}(x(b \lambda))\right)^{-1 / 2} \int_{x(\lambda)}^{x(b \lambda)}(x-x(\lambda))^{-1 / 2} d x \\
\left.\quad=2 x(\lambda)^{-2}\left(-\frac{d U}{d x}(x(b \lambda))\right)^{-1 / 2}(x(b \lambda))-x(\lambda)\right)^{1 / 2} \\
\quad \leqq C\left(-x(b \lambda)^{2} \frac{d U}{d x}(x(b \lambda))\right)^{-1 / 2} x(b \lambda)^{-1 / 2} .
\end{aligned}
$$

Applying Lemma 4.1, we see that the last term tends to zero as $\lambda \rightarrow 0$, which implies $I_{2}=o(1)$ as $\lambda \rightarrow 0$. This completes the proof.

Here we give the estimates of the solution of the equation $(4 \cdot 2)$.

Lemma 4. 4. (The estimate for $x \geqq x(\lambda)$.) Let $g(x)$ be a solution of $(4 \cdot 2)$. Then we have for $x \geqq x(\lambda)$

$$
\begin{gathered}
|g(x)| \leqq C e^{C A(x)} \\
\left|g(x)-\eta_{0}(\zeta(x))\right| \leqq C\left(e^{C_{A}(x)}-1\right),
\end{gathered}
$$

where $A(x)=\int_{x}^{\infty}\left|Q(y)(\lambda-U(y))^{1 / 2}\right| d y$ and $C$ is a constant independent 
of $\lambda, x$.

Proof. Since we have the estimates (4.3) and (4.4), the expected estimates may be obtained by ordinary calculations, so we omit the proof.

Put

$$
B(x)=\int_{x}^{x(\lambda)}\left|Q(y)(\lambda-U(y))^{1 / 2}\right| d y
$$

and let $h(x)$ be a unique solution of the integral equation

$$
h(x)=h_{0}(x)-\int_{x}^{\infty} G_{0}(x, y) Q_{0}(y) h(y)(-U(y))^{1 / 2} d y,
$$

where

$$
\left\{\begin{array}{l}
Q_{0}(x)=-\frac{1}{4} \frac{d^{2} U / d x^{2}}{U(x)^{2}}+\frac{5}{16} \frac{(d U / d x)^{2}}{U(x)^{3}}, \\
h_{0}(x)=2^{1 / 2} \exp \left(\frac{2 \nu+1}{4} \pi i-\int_{0}^{x}(U(y))^{1 / 2} d y\right), \\
G_{0}(x, y)=\frac{1}{2 i}\left\{\exp \left(-\int_{x}^{y}(U(u))^{1 / 2} d u\right)-\exp \left(\int_{x}^{y}(U(u))^{1 / 2} d u\right)\right\}, \text { for } y \geqq x .
\end{array}\right.
$$

Lemma 4. 5. (The estimate for $0<x \leqq x(\lambda)$.) Under the condition (4.6), $g(x)$ has the estimate

$$
|g(x)| \leqq C \exp (|\zeta(x)|+C B(x))
$$

for every $0<x \leqq x(\lambda)$ and $0<\lambda \leqq 1$, moreover $h(x, \lambda)=e^{-|\zeta(0)|} g(x)$ tends to $h(x)$ as $\lambda \rightarrow 0$.

Proof. Let

$$
\left\{\begin{array}{l}
\alpha=\frac{1}{4 i} \int_{x(\lambda)}^{\infty} \eta_{1}(\zeta(y)) g(y) Q(y)(\lambda-U(y))^{1 / 2} d y \\
\beta=-\frac{1}{4 i} \int_{x(\lambda)}^{\infty} \eta_{0}(\zeta(y)) g(y) Q(y)(\lambda-U(y))^{1 / 2} d y
\end{array}\right.
$$

Then by the definition of $g$ we have for $0<x \leqq x(\lambda)$ 


$$
\begin{aligned}
g(x)= & (1+\alpha) \eta_{0}(\zeta(x))+\beta \eta_{1}(\zeta(x)) \\
& -\int_{x}^{x(\lambda)} G(\zeta(x), \zeta(y)) Q(y) g(y)(\lambda-U(y))^{1 / 2} d y .
\end{aligned}
$$

Put

$$
\xi_{0}(\zeta)=(1+\alpha) \eta_{0}(\zeta)+\beta \eta_{1}(\zeta)
$$

Then $g$ satisfies the equation

$(4 \cdot 16) \quad g(x)=\xi_{0}(\zeta(x))$

$$
-\int_{x}^{x(\lambda)} G(\zeta(x), \zeta(y)) Q(y) g(y)(\lambda-U(y))^{1 / 2} d y .
$$

By Lemma 4.2, we have the estimate

$$
|\alpha|+|\beta| \leqq C\left(e^{C A(x(\lambda))}-1\right) .
$$

Lemma 4.3 implies $A(x(\lambda))=o(1)$ as $\lambda \rightarrow 0$, whence by $(4 \cdot 17)$ we obtain

$$
\alpha=o(1), \beta=o(1) \text { as } \lambda \rightarrow 0 \text {. }
$$

This together with $(4 \cdot 3)$ gives the estimate

$$
\left|\xi_{0}(\zeta(x))\right| \leqq C e^{|\zeta(x)|}
$$

for $0<\lambda \leqq 1$ and $0<x \leqq x(\lambda)$. Put

$$
\left\{\begin{array}{l}
g_{0}(x)=\xi_{0}(\zeta(x)), \\
g_{n}(x)=-\int_{x}^{x(\lambda)} G(\zeta(x), \zeta(y)) Q(y) g_{n-1}(y)(\lambda-U(y))^{1 / 2} d y .
\end{array}\right.
$$

Then using $(4 \cdot 5)$ and $(4 \cdot 19)$, we obtain

$$
\left|g_{n}(x)\right| \leqq C \frac{(C B(x))^{n}}{n !} e^{|\zeta(x)|},
$$

which gives the estimate $(4 \cdot 15)$.

Now we proceed to the proof of the latter half of the lemma. Put

$$
\left\{\begin{array}{l}
k_{n}(x)=e^{-|\zeta(0)|} g_{n}(x), \\
h_{n}(x)=-\int_{x}^{\infty} G_{0}(x, y) h_{n-1}(y) Q_{0}(y)(-U(y))^{1 / 2} d y .
\end{array}\right.
$$

We must show that each $k_{n}$ tends to $h_{n}$ as $\lambda \rightarrow 0$. First we see by $(4 \cdot 18)$ and $(4 \cdot 3) k_{0}(x) \rightarrow h_{0}(x)$ as $\lambda \rightarrow 0$. Assume $k_{n} \rightarrow h_{n}$ as $\lambda \rightarrow 0$. By the definition of $k_{n}$, we have 


$$
k_{n+1}(x)=I_{n}+J_{n}
$$

where

$$
\left\{\begin{array}{l}
I_{n}=-\int_{x(a \lambda)}^{x(\lambda)} G(\zeta(x), \zeta(y)) k_{n}(y) Q(y)(\lambda-U(y))^{1 / 2} d y \\
J_{n}=-\int_{x}^{x(a \lambda)} G(\zeta(x), \zeta(y)) k_{n}(y) Q(y)(\lambda-U(y))^{1 / 2} d y .
\end{array}\right.
$$

From $(4 \cdot 20)$ we have the inequality

$$
\left|I_{n}\right| \leqq \frac{(C B(x(a \lambda)))^{n+1}}{(n+1) !} \exp \left(\int_{0}^{x}(U(y)-\lambda)^{1 / 2} d y\right) .
$$

which together with Lemma 4.3 gives the estimate

$$
I_{n}=o(1) \text { as } \lambda \rightarrow 0 \text {. }
$$

Here remember the definition of $Q$ :

$$
Q(x)=-\frac{1}{4} \frac{d^{2} U / d x^{2}}{(\lambda-U(x))^{2}}-\frac{5}{16} \frac{(d U / d x)^{2}}{(\lambda-U(x))^{3}}+\frac{5}{36} \frac{1}{\zeta(x)^{2}} .
$$

In the estimate for $J_{n}$, the last term is negligible. For we have

$$
\begin{aligned}
& \int_{x}^{x(a \lambda)}\left|G(\zeta(x), \zeta(y)) k_{n}(y)(\lambda-U(y))^{1 / 2} \zeta(y)^{-2}\right| d y \\
& \quad \leqq \frac{C}{n !} \exp \left(\int_{0}^{x}(U(y)-\lambda)^{1 / 2} d y\right) \int_{x}^{x(a \lambda)}(C B(y))^{n}|\zeta(y)|^{-2}(-d|\zeta(y)|) \\
& \quad \leqq C \frac{(C B(x))^{n}}{n !}|\zeta(x(a \lambda))|^{-1} \exp \left(\int_{0}^{x}(U(y)-\lambda)^{1 / 2} d y\right)
\end{aligned}
$$

as $\lambda \rightarrow 0$ from Lemmas 4.2 and 4.3. Noting the inequality for $0<y \leqq x(a \lambda)$

$$
U(y)-\lambda=U(y)-a^{-1} U(x(a \lambda)) \geqq U(y)\left(1-a^{-1}\right),
$$

we have

$$
(U(y))^{1 / 2}\left|Q(y)-\frac{5}{36} \zeta(y)^{-2}\right| \leqq C\left(\frac{d^{2} U / d y^{2}}{U(y)^{2}}+\frac{(d U / d y)^{2}}{U(y)^{3}}\right)(U(y))^{1 / 2} .
$$

(4.12) implies that the right hand side is integrable in each $(c, \infty)$, whence applying the dominated convergence theorem and noting $(4 \cdot 21)$ we see that $k_{n+1}(x)$ converges to $h_{n+1}(x)$ as $\lambda \rightarrow 0$ for each $x>0$. Consequently we see $k_{n} \rightarrow h_{n}$ as $\lambda \rightarrow 0$ for each $n$. On the other hand from Lemma 4.3 and $(4 \cdot 20)$ we have the estimate 


$$
\left|k_{n}(x)\right| \leqq \frac{C^{n+1}}{n !} \exp \left(-\int_{0}^{x}(U(y)-\lambda)^{1 / 2} d y\right)
$$

for every $x>0$ and $0<\lambda \leqq 1$. Then it is easy to see that $h(x, \lambda)=\sum_{n=0}^{\infty} k_{n}(x)$ tends to $h(x)=\sum_{n=0}^{\infty} h_{n}(x)$, which proves the lemma.

Define $f(x, \lambda)$ by

$$
f(x, \lambda)=\frac{g(x, \lambda)}{2^{1 / 2}(\lambda-U(x))^{1 / 4}} \exp \left(-\frac{2 \nu+1}{4} \pi i-|\zeta(0)|\right),
$$

where $g(x, \lambda)=g(x)$. Then as we stated before, $f(x, \lambda)$ satisfies the equation

$$
\frac{d^{2}}{d x^{2}} f(x, \lambda)=(-\lambda+U(x)) f(x, \lambda)
$$

Lemma 4. 6. $f(0,0+) \neq 0$ and $f(x, 0+)$ satisfies the equation

$$
\frac{d^{2}}{d x^{2}} f(x, 0+)=U(x) f(x, 0+) .
$$

Proof. Fix $a>0$. Let $\varphi(x, \lambda), \psi(x, \lambda)$ be the solutions of $(4 \cdot 1)$ satisfying the initial conditions

$$
\begin{cases}\varphi(a, \lambda)=1, & \psi(a, \lambda)=0, \\ \frac{d}{d x} \varphi(a, \lambda)=0, & \frac{d}{d x} \psi(a, \lambda)=1 .\end{cases}
$$

Since from the assumption $\int^{+\infty} \log u n(d u)<\infty, U(x)$ is integrable in the neighbourhood of the origin, $\varphi$ and $\psi$ are continuous with respect to the variable $(x, \lambda) \in[0, \infty) \times \mathbb{R}^{1}$. Note the identity

$$
f(x, \lambda)=f(a, \lambda) \varphi(x, \lambda)+\frac{d}{d x} f(a, \lambda) \psi(x, \lambda)
$$

for any $x \geqq 0, \lambda \in \boldsymbol{R}^{1}$. Lemma 4.5 implies the existence of $f(x, 0+)$ for any $x>0$. Observing $\psi(x, 0) \neq 0$, we see by $(4 \cdot 23)$ that $\frac{d}{d x} f(a, 0+)$ also exists. Therefore it is evident from $(4 \cdot 23)$ that $f(x, 0+)$ exists for any $x \geqq 0$ and has the form:

$$
f(x, 0+)=f(a, 0+) \varphi(x, 0)+\frac{d}{d x} f(a, 0+) \psi(x, 0) .
$$


This implies that $f(x, 0+)$ is a solution of the equation $\frac{d^{2}}{d x^{2}} f(x)=U(x)$ $\cdot f(x)$. By $(4 \cdot 14) f(x, 0+)$ has the estimate

$$
|f(x, 0+)| \leqq C U(x)^{-1 / 4} \exp \left(-\int_{0}^{x}(U(y))^{1 / 2} d y\right) .
$$

Noting the inequality

$$
U(x)^{1 / 4} \exp \left(\int_{0}^{x}(U(y))^{1 / 2} d y\right) \geqq U(x)^{1 / 4} \int_{0}^{x}(U(y))^{1 / 2} d y \geqq U(x)^{3 / 4} x,
$$

we see by Lemma 4.1 that the right hand side of $(4 \cdot 24)$ tends to zero as $x \rightarrow \infty$, whence we have

$$
f(x, 0+) \rightarrow 0 \text { as } x \rightarrow+\infty .
$$

Suppose $f(0,0+)=0$. Then $f(x, 0+)$ satisfies the equation

$$
f(x, 0+)=\frac{d}{d x} f(0,0+) x+\int_{0}^{x}(x-y) f(y, 0+) U(y) d y .
$$

Noting $U(y)>0$ and $\frac{d}{d x} f(0,0+) \neq 0$, we have $|f(x, 0+)| \geqq\left|\frac{d}{d x} f(0,0+)\right| x$ for $x>0$, which contradicts $(4 \cdot 25)$. Thus we complete the proof.

With the help of these lemmas we obtain the main

Theorem 4. 7. Suppose $U(x)=\frac{1}{x} \int_{0}^{\infty}\left(1-e^{-x u}\right) n(d u)$ satisfies

$$
\int_{0+} U(x) d x<\infty, \quad \frac{-x d U / d x}{U(x)} \geqq c>0
$$

for every sufficiently large $x$. Then the spectral distribution $N(\lambda)$ has the asymptotic behaviour:

$$
N(\lambda) \sim \frac{1}{\pi|f(0)|^{2}} \exp \left(-2 \int_{0}^{x(\lambda)}(U(y)-\lambda)^{1 / 2} d y\right) \text { as } \lambda \rightarrow 0
$$

where $f$ is a unique solution of the equation

$$
\begin{array}{r}
\frac{d^{2}}{d x^{2}} f(x)=U(x) f(x), \quad f(x) \sim U(x)^{-1 / 4} \exp \left(-\int_{0}^{x}(U(y))^{1 / 2} d y\right) \\
\text { as } x \rightarrow+\infty,
\end{array}
$$


and $x(\lambda)$ is the inverse function of $U(x)$.

Proof. All we have to do is to find the relation between $f(x, \lambda)$ and $f_{-}(x, \lambda)$ of Theorem 3.2. Noting $A(x)=o(1)$ as $x \rightarrow \infty$, we have by Lemma 4 . 4 ,

$$
\begin{aligned}
f(x, \lambda) & \sim \frac{\eta_{0}(\zeta(x))}{2^{1 / 2}(\lambda-U(x))^{1 / 4}} \exp \left(-\frac{2 \nu+1}{4} \pi i-|\zeta(0)|\right) \\
& \sim \lambda^{-1 / 4} \exp (-i \zeta(x)-|\zeta(0)|)
\end{aligned}
$$

as $x \rightarrow \infty$. Therefore by the definition of $f_{-}(x, \lambda)$ we see

$$
f_{-}(x, \lambda)=\lambda^{1 / 4} e^{|\zeta(0)|} f(x, \lambda) .
$$

Hence Theorem 3.2 together with Lemma 4.6 gives us

$$
\begin{aligned}
N(\lambda) & =\frac{\lambda^{1 / 2}}{\pi\left|f_{-}(0, \lambda)\right|^{2}}=\frac{e^{-2|\zeta(0)|}}{\pi|f(0, \lambda)|^{2}} \\
& \sim \frac{1}{\pi|f(0,0+)|^{2}} \exp \left(-2 \int_{0}^{x(\lambda)}(U(y)-\lambda)^{1 / 2} d y\right)
\end{aligned}
$$

as $\lambda \rightarrow 0$. This complete the proof.

Corollary 4.8. Suppose $Q$ is a stable process with index $\alpha$, namely $U(x)=n x^{-(1-\alpha)}(0<\alpha<1)$. Then we have the asymptotic form:

$$
N(\lambda) \sim B_{\alpha} n^{\frac{1}{1+\alpha}} \exp \left(-C_{\alpha} n^{\frac{1}{1}-\alpha} \lambda^{-\frac{1}{1}-\bar{\alpha}+\frac{1}{2}}\right)
$$

where

$$
B_{\alpha}=(1+\alpha)^{-\frac{1-\alpha}{1+\alpha}} \Gamma\left(\frac{1}{1+\alpha}\right)^{-2}, \quad C_{\alpha}=\pi^{1 / 2} \Gamma\left(\frac{1}{1-\alpha}-\frac{1}{2}\right) \Gamma\left(\frac{1}{1-\alpha}\right)^{-1} .
$$

Proof. Note $2 \int_{0}^{x(\lambda)}(U(y)-\lambda)^{1 / 2} d y=C_{\alpha} n^{\frac{1}{1-a} \lambda^{-\frac{1+\alpha}{2}(1-\alpha)}}$ and

$$
\left\{\begin{array}{l}
f(x)=2(\pi(1+\alpha))^{-1 / 2} x^{1 / 2} K \frac{1}{1+\alpha}\left(\frac{2 n^{1 / 2}}{1+\alpha} x^{(1+\alpha) / 2}\right) \\
f(0)=\pi^{1 / 2}(1+\alpha)^{(1-\alpha) / 2(1+\alpha)} n^{-1 / 2(1+\alpha)} \Gamma\left(\frac{1}{1+\alpha}\right),
\end{array}\right.
$$

where $K_{\nu}$ is the modified Bessel function, which proves the corollary. 
The above system is a continuous analogue of the one considered by M. Fukushima [14] and the result gives us some suggestions to a discrete system.

Corollary 4.9. Suppose

$$
\int_{0}^{\infty} n(d u)+\int^{+\infty} \log u n(d u)<\infty .
$$

Then

$$
N(\lambda) \sim \frac{1}{|f(0)|^{2}} \exp \left(-2 \int_{0}^{x(\lambda)}(U(y)-\lambda)^{1 / 2} d y\right)
$$

as $\lambda \rightarrow 0$, and further we have

$$
2 \int_{0}^{x(\lambda)}(U(y)-\lambda)^{1 / 2} d y=n \pi \lambda^{-1 / 2}+o\left(\lambda^{-1 / 2}\right)
$$

where $n=\int_{0}^{\infty} n(d u)$.

Proof. In this case the second condition of Theorem 4.7 is satisfied automatically in view of Lemma 4.1. The estimate $(4 \cdot 27)$ may be verified without difficulty.

Remark. When $Q$ is a Poisson process, we may obtain a more explicit asymptotic form:

$$
N(\lambda) \sim \frac{1}{|f(0)|^{2}} e^{-n \pi \lambda-1 / 2} .
$$

This is a completion of the result of T. P. Eggarter [6]. We remark that S. Nakao [9] obtained the estimate $\lambda^{1 / 2} \log N(\lambda) \rightarrow-n \pi$ by the different method from ours.

Although it is more desirable to consider this problem for general additive process, we have not succeeded yet. However we remark that M.Fukushima and S.Nakao [8] treated the white Gaussian noise potential and gave a precise formulation of the eigenvalue problem to obtain the explicit form of $N(\lambda)$. 


\section{§5. A Remark to the Spectral Distribution Function of a One Dimensional Hamiltonian with a Random Weight}

In this section we treat an operator

$$
L=-\frac{d}{d M} \frac{d}{d x}
$$

where $\{M(x)\}$ is a increasing process of stationary independent increments. We study the spectral distribution function of $L$. Horvever the method is similar to the one of $\S 4$, we avoid going into details.

Let $\psi$ be the exponent of the characteristic function of $M$, namely

$$
E\left(e^{-\xi M(x)}\right)=e^{-x \psi(\xi)},
$$

and suppose that $\psi$ has the form:

$$
\psi(\xi)=a \xi+\int_{0}^{\infty}\left(1-e^{-\xi u}\right) n(d u),
$$

where $a \geq 0$ and $\int_{0}^{\infty} \min (1, u) n(d u)<\infty$. First we consider the case $a>0$. Define an additive process $M_{0}$ with the exponent $\int_{0}^{\infty}\left(1-e^{-\hat{\xi} u}\right) n(d u)$ by the equation

$$
M(x)=a x+M_{0}(x) .
$$

Let $(\xi(x), \eta(x))$ be the solutions of the equation

$$
\left\{\begin{array}{l}
d \xi(x)=\eta(x) d x \\
d \eta(x)=-\lambda a \xi(x) d x-\lambda \xi(x) d M_{0}(x) .
\end{array}\right.
$$

Then Corollary 1. 12 gives us the identity

$$
N(\lambda,[0, l])=\#\{x \in[0, l] ; \xi(x)=0\}+\varepsilon(\lambda),
$$

where $|\varepsilon(\lambda)| \leqq 2$. Regarding $\lambda$ as $\lambda a$ and $Q$ as $-\lambda M_{0}$ in (2.1) of $\S 2$, we may study the spectral distribution of $L$ in the same way as in the previous section if we perform the analytic continuation of the solution of the Frisch-Lloyd formula to the lower half plane. Namely we have

$$
N(\lambda)=\frac{\lambda^{1 / 2}}{\pi|f(0,1 / \lambda)|^{2}}
$$

where $f(x, \lambda)$ is a unique solution of the equation 


$$
\left\{\begin{array}{l}
\frac{d^{2}}{d x^{2}} f(x, \lambda)=-\lambda U(x) f(x, \lambda), \\
f(x, \lambda) \sim U(x)^{-1 / 4} \exp \left(i \lambda^{1 / 2} \int_{0}^{x}(U(y))^{1 / 2} d y\right),
\end{array}\right.
$$

as $x \rightarrow \infty$, where $U(x)=a+\frac{1}{x} \int_{0}^{\infty}\left(1-e^{-x u}\right) n(d u)$.

Letting $a \rightarrow 0$ in the equation (5.2), we see that the solutions $f(x, \lambda)$ converges to the solution of the equation $(5 \cdot 2)$ regarding $a$ as 0 . Hence it may be concluded that the process $\{z(x)\}$ is ergodic even in the case $a=0$. Summing up these arguments we obtain

Theorem 5. $\mathbb{1}$. Suppose $U(x)$ satisfies

$$
\int_{0+} U(x) d x<\infty .
$$

Then the spectral distribution $N(\lambda)$ may be expressed as (5.1) by the solution of $(5 \cdot 2)$.

Example 直 $a=0, U(x)=n x^{-(1-\alpha)} \quad(0<\alpha<1)$.

$$
N(\lambda)=n^{1 / 1+\alpha} \Gamma\left(\frac{1}{1+\alpha}\right)^{-2}(1+\alpha)^{-(1-\alpha) /(1+\alpha)} \lambda^{\alpha /(1+\alpha)} .
$$

Example 2. $a=0, U(x)=\frac{n}{x+c}(c>0)$, namely $d n(x)=c n e^{-c x} d x$.

$$
N(\lambda)=\frac{\lambda}{c \pi^{2}}\left|H_{1}^{(1)}\left(2(n c / \lambda)^{1 / 2}\right)\right|^{-2},
$$

where $H_{1}^{(1)}$ is the Hankel function of the first kind.

\section{§6. A Remark to the Spectral Distribution of Equations Defined on the Whole Line $\mathbb{R}^{1}$}

In the previous section we have considered the case when the equations are defined on the half line. In this section we remark a relation between the spectral function on the half line and the one on the whole line.

Let $L$ be one of the operators 


$$
-\frac{d \frac{d}{d x}-d Q}{d x},-\frac{d}{d M} \frac{d}{d x},
$$

where $Q$ is a function of bounded variation in each finite interval of $\mathbb{R}^{1}$ and $M$ is a nondecreasing function on $\boldsymbol{R}^{1}$. Put

$$
\left\{\begin{array}{l}
N_{+}(\lambda)=\lim _{l \rightarrow \infty} \frac{1}{l} N(\lambda,[0, l]), \\
N_{-}(\lambda)=\lim _{l \rightarrow \infty} \frac{1}{l} N(\lambda,[-l, 0]), \\
N(\lambda)=\lim _{l \rightarrow \infty} \frac{1}{2 l} N(\lambda,[-l, l]),
\end{array}\right.
$$

if they exist. Then we have the following

Theorem 6. 1. Suppose $N_{ \pm}(\lambda)$ exists for each $\lambda$. Then $N(\lambda)$ also exists and has the form:

$$
N(\lambda)=\frac{1}{2}\left(N_{+}(\lambda)+N_{-}(\lambda)\right)
$$

Proof. Let $N_{0}(\lambda,[a, b])$ and $N_{\infty}(\lambda,[a, b])$ be the number of eigenvalues not exceeding $\lambda$ for the boundary value problem

$$
f(a)=f(b)=0 \quad \text { and } \quad \frac{d f}{d x}(a)=\frac{d f}{d x}(b)=0,
$$

respectively. Then these functions satisfy the inequalities

$$
\left\{\begin{array}{l}
0 \leqq N_{\infty}(\lambda,[a, b])-N_{0}(\lambda,[a, b]) \leqq 2, \\
N_{0}(\lambda,[a, b]) \leqq N(\lambda,[a, b]) \leqq N_{\infty}(\lambda,[a, b]),
\end{array}\right.
$$

by the results of $\S 1$. Further the mini-max principle gives the inequalities for $a<c<b$

$$
\left\{\begin{array}{l}
N_{\infty}(\lambda,[a, b]) \leqq N_{\infty}(\lambda,[a, c])+N_{\infty}(\lambda,[c, b]), \\
N_{0}(\lambda,[a, b]) \geqq N_{0}(\lambda,[a, c])+N_{0}(\lambda,[c, b]) .
\end{array}\right.
$$

Then the theorem is easily obtained.

Owing to this theorem, we may apply the results of $\S 4 \sim 5$ to the 
spectral distribution of equations on the whole line.

\section{References}

[1] Lax, M. and Phillips, J. C., One-dimensional impurity bands, Phys. Rev., 110 (1958), 41-49.

[2] Lifšic, I. M., Energy spectrum structure and quantum states of disordered condensed systems, Soviet Phys. Uspekhi, 7 (1965), 549-573.

[3] Frisch, H. L. and Lloyd, S. P., Electron levels in one-dimensional lattice, Phys. Rev., 120 (1960), 1175-1189.

[4] Benderskii, M. M. and Pastur, L. A., On the spectrum of the one-dimensional Schrödinger equation with a random potential, Mat. Sb., 82 (1970), 273-284.

[5] Calculation of the average number of states in a model problem, Soviet Phys. JETP, 30 (1970), 158-162.

[6] Eggarter, T. P., Some exact results on electron energy levels in certain one-dimensional random potentials, Phys. Rev., B, 5 (1972), 3863-3865.

[7] Pastur, L. A., Spectra of random self-adjoint operator, Russian Math. Surveys, 28 (1973), 1-69.

[8] Fukushima, M. and Nakao, S., On spectra of the Schrödinger operator with a white Gaussian noise potential, to appear in Z. Wahrscheinlichkeitstheorie verw. Gebiete.

[9] Nakao, S., On the spectral distribution of the Schrödinger operator with random potential, to appear.

[10] Kac, I. S. and Krein, M. G., On spectral functions of a string, Amer. Math. Soc. Transl. (2), 103 (1974), 19-102.

[11] Levin, B. Ja., Distribution of zeros of entire functions, Transl. Math. Monographs 5, Amer. Math. Soc., Providence, R. I., 1964.

[12] Bellman, R., Stability theory of differential equations, Dover Publications, Inc. New York, 1953.

[13] Titchmarsh, E. C., Eigenfunction expansions, part 2, Oxford, 1962.

[14] Fukushima, M., On the spectral distribution of a disordered system and the range of a random walk, Osaka J. Math., 11 (1974), 73-85. 